\title{
Finiteness in derived categories of local rings
}

\author{
W. Dwyer, J. P. C. Greenlees and S. Iyengar*
}

\begin{abstract}
New homotopy invariant finiteness conditions on modules over commutative rings are introduced, and their properties are studied systematically. A number of finiteness results for classical homological invariants like flat dimension, injective dimension, and Gorenstein dimension, are established. It is proved that these specialize to give results concerning modules over complete intersection local rings. A noteworthy feature is the use of techniques based on thick subcategories of derived categories.
\end{abstract}

Mathematics Subject Classification (2000). Primary 13D05, 13D99; Secondary 18E30, $18 \mathrm{G} 99$.

Keywords. Complete intersections, Gorenstein rings, homological dimensions, perfect complexes, thick subcategories.

\section{Contents}

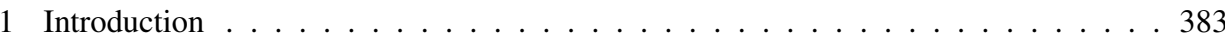

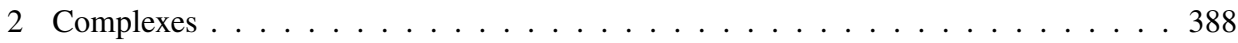

3 Thick subcategories of derived categories . . . . . . . . . . . . . . . 391

4 Virtually small complexes . . . . . . . . . . . . . . . . . . . . . . . . . . . . . . . . . . . . . . . . . . . . . . . . .

5 Virtually small complexes as test objects . . . . . . . . . . . . . . . . 405

6 Gorenstein dimensions . . . . . . . . . . . . . . . . . . . . . . 411

7 Descent . . . . . . . . . . . . . . . . . . . . 415

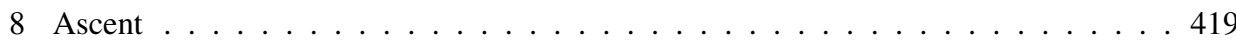

9 Complete intersection local rings . . . . . . . . . . . . . . . . . . . . . 424

\section{Introduction}

In this paper we shall investigate several homotopy invariant finiteness conditions on modules and complexes over commutative noetherian rings. This leads to new results in classical commutative algebra, some of which are discussed in the introduction. First, we describe our philosophy.

\footnotetext{
*S. I. was partly supported by the NSF under grant DMS 0442242.
} 
Our earlier work [13] transplants ideas from commutative algebra into a homotopy theoretic context. The process is conceptually illuminating since it shows that several classical duality theorems in algebra and topology are not just analogous but actually manifestations of the same phenomenon. However, the main justification for the approach is that it leads to interesting and concrete new results in homotopy theory. In the present paper we reverse the process, and use ideas inspired by homotopy theory to solve problems in ring theory.

Before one can apply a concept from algebra in the topological context one has to express it in a homotopy invariant form. The idea of formulating statements about a ring in terms of its derived category is a familiar one, but because the framework of [13] was more general than that, we were forced to concentrate on the crudest and most robust properties. It is striking that these give new insights even in commutative ring theory. The homological characterization of regular local rings illustrates this point.

A local ring $R$ is said to be regular if its maximal ideal $\mathfrak{m}$ is generated by a regular sequence. Auslander, Buchsbaum, and Serre proved that $R$ is regular if and only if the residue field $k$ has finite projective dimension. Since the finiteness of the projective dimension of a homologically finite complex $M$ is detected by $\operatorname{Ext}_{R}^{*}(M, k)$, regularity is detected at the level of derived categories. However, one can reformulate it in structural terms: $k$ has finite projective dimension precisely when it is equivalent in the derived category to a perfect complex, that is to say, a finite complex consisting of finitely generated projectives. Furthermore, perfectness can be captured entirely categorically: $M$ is perfect if and only if it is small, in the sense that $\mathbf{R H o m}_{R}(M,-)$ preserves arbitrary direct sums in the derived category. Thus one reaches the homotopy invariant formulation: $R$ is regular if and only if $k$ is small in the derived category. The selection of the particular module $k$ is unnecessary: $R$ is regular if and only if every homologically finite complex $M$ is small in the derived category. The advantages of the homological formulation are well known: for example, it allows one to prove that localizations of regular local rings are regular. The structural formulation shows that regularity is preserved by equivalences of derived categories, and makes sense even when one has no notion of elements or of Ext.

Small complexes remain important in commutative ring theory, but the focus of our work is on virtually small complexes: those complexes $M$ with the much weaker property that either $\mathrm{H}(M)=0$, or the thick subcategory generated by $M$ contains a non-trivial small complex. A detailed explanation of the terms involved in the preceding definition, including some background on thick subcategories, is provided in Section 3. We study also the closely related proxy small complexes, which played a crucial role in [13] and provided the original motivation for the present investigations.

Evidently every small complex is virtually small. However there are many more virtually small complexes than small ones; for instance, if $M$ is virtually small, then 
so is $M \oplus X$, for any complex $X$ of $R$-modules, as long as $\mathrm{H}(M) \neq 0$. A better illustration is the result below, which contains parts of Theorems 4.9 and 7.7. Given a complex $N$ of $S$-modules, we say $\mathrm{H}(N)$ is finite (respectively, artinian) if the $S$-module $\mathrm{H}_{i}(N)$ is finite (respectively, artinian) for each $i$, and zero for $|i| \gg 0$.

Theorem I. Let $\varphi: R \rightarrow S$ be a local homomorphism and $N$ a complex of $S$-modules with $\mathrm{H}(N)$ either finite or artinian. If either $\operatorname{fd}_{R} N$ is finite or $S$ is regular, then $N$ is virtually small over $R$.

Specializing $\varphi$ to the surjective homomorphism $R \rightarrow k$ and $N$ to $k$, we see that the residue field $k$ is virtually small over $R$; alternatively, it is not hard to check that the Koszul complex of $R$ is in the thick category generated by $k$. This may be a little unsettling: from the classical point of view, exemplified by the AuslanderBuchsbaum-Serre theorem, the homological properties of $k$ differ drastically from those of small complexes. Nonetheless, virtually small complexes are very useful as test objects for finiteness of homological invariants. Sections 5 and 6 establish a number of results to validate this claim. We now provide some illustrations.

Let $\psi: Q \rightarrow R$ be a local homomorphism and $M$ a complex of $R$-modules, which is viewed as a complex of $Q$-modules via $\psi$. It is classical that when $M$ is small many homological properties of $R$ over $Q$ are inherited by $M$; for example, it follows from a result of Cartan-Eilenberg [9] that, under this condition on $M$, if $\mathrm{fd}_{Q} R$ is finite, then so is $\operatorname{fd}_{Q} M$. Recent research, notably that of Apassov [1], Foxby and Iyengar [15], and Iyengar and Sather-Wagstaff [20], has uncovered certain situations when the converse is true. The main discovery of this work is these are instances of the same phenomenon, which is valid in much greater generality, and even when $M$ is only assumed to be virtually small.

For example we may consider flat dimension. As a converse to the result of CartanEilenberg recalled above, Foxby and Iyengar [15] prove that when $M$ is small, $\mathrm{fd}_{Q} M$ finite implies $\mathrm{fd}_{Q} R$ finite. On the other hand, if $\mathrm{fd}_{Q} k$ is finite, then $Q$ is regular, and hence $\operatorname{fd}_{Q} R$ is finite; here one is using the Auslander-Buchsbaum-Serre theorem. We generalize these results as follows:

Theorem II. Let $M$ be a virtually small complex of $R$-modules with $\mathrm{H}(M) \neq 0$. If $\mathrm{fd}_{Q} M$ is finite, then $\mathrm{fd}_{Q} R$ is finite as well.

The proof in [15] proof is involved, and based on properties of depth for complexes. In contrast, the one given here is straightforward; it is based on work of Hopkins [18] and Neeman [24] on thick subcategories of derived categories of commutative noetherian rings. It was this argument, which is crystallized in 5.2, that convinced us of the relevance of virtually small complexes to classical commutative ring theory. 
We prove numerous other results that demonstrate the efficacy of this technique: Theorems 5.3 and 6.6 have the same flavour as the one above, and deal with injective dimension and Gorenstein dimension, respectively. Theorem 5.10 concerns asymptotic invariants, and says that the complexity and curvature of $R$ (over $\psi$ ) are smaller than those for any virtually small complex. In a different direction, Theorem 5.12 states that if for a complex $Y$ of $R$-modules with $\mathrm{H}(Y)$ degreewise finite, the amplitude of $M \otimes_{R}^{\mathbf{L}} Y$ is finite for a virtually small complex $M$ with $\mathrm{H}(M) \neq 0$, then the amplitude of $Y$ is finite.

With the importance of virtual smallness established, Sections 7 and 8 describe other sources of virtually small complexes and investigate their behaviour under standard constructions: virtual smallness is much more robust than smallness.

Not everything homologically finite is virtually small. One example is described in 5.8, but it is difficult to decide when a complex, even a homologically finite one, is virtually small. This is in contrast with the case of small complexes where methods are implemented in computer algebra packages such as Macaulay. It is therefore natural to ask: over which local rings are all homologically finite complexes of modules virtually small?

Our principal result in this direction is Theorem 9.4, part of which reads:

Theorem III. If a local ring $R$ is a complete intersection, then any complex of $R$ modules which is homologically either finite or artinian is virtually small.

Section 9 also contains the necessary background on complete intersection rings. In view of this theorem, all the results in Sections 5 and 6 specialize to the case where $R$ is a complete intersection. We mention one, which is part of Theorem 9.7. It extends, and its proof provides a different perspective on, $[1,(\mathrm{R}, \mathrm{CI})]$, which treats the special case where $M$ is a finite $R$-module. Note that, with $M=R$ one recovers the well-known result that both regularity and the complete intersection property descend along homomorphisms of finite flat dimension.

Corollary IV. Let $Q \rightarrow R$ be a local homomorphism and $M$ a complex of $R$-modules with $\mathrm{H}(M) \neq 0$ either finite or artinian. If $R$ is a complete intersection and $\operatorname{fd}_{Q} M$ is finite, then $Q$ is a complete intersection and codim $Q \leq \operatorname{codim} R$.

Crucial in the proof of Theorem III are stability properties of virtual smallness. We consider a ring homomorphism $\varphi: R \rightarrow S$ and virtual smallness over $R$ and over $S$ for complexes of $R$-modules. Following the variance on prime spectra, we say that virtual smallness descends along $\varphi$ if each complex of $S$-modules that is virtually small over $S$ is also virtually small over $R$. Most of our discussion on descent is in Section 7, and the following excerpt from Theorem 7.3 gives a flavour of some of the results found there. 
Theorem V. Let $\varphi: R \rightarrow S$ be a surjective homomorphism of noetherian rings. If $S$ is virtually small as an R-module then virtual smallness descends along $\varphi$.

Another noteworthy situation when virtual smallness descends is the case when $S$ is regular and module finite over $R$. In Section 8, we turn to ascent: virtual smallness ascends along $\psi: Q \rightarrow R$ if any complex of $R$-modules that is virtually small over $Q$ is virtually small over $R$. Ascent is a lot more delicate than descent, and our main result dealing with this issue is as follows:

Theorem VI. Let $\psi: Q \rightarrow R$ be a homomorphism of rings. If $R \otimes_{Q}^{\mathbf{L}} R$ can be finitely built from $R$ as a bimodule, then virtual smallness ascends along $\psi$.

This criterion for ascent is part of Theorem 8.3, see also 8.10, and it is a crucial ingredient in our proof of Theorem III. The statement itself is explained in detail in Section 8. One reason for quoting it here is to point out that it is reminiscent of the classical Hochschild-Kostant-Rosenberg criterion for smoothness: a homomorphism $\psi$ essentially of finite type is smooth if and only if it is flat and the $\left(R \otimes_{Q} R\right)$-module $R$ is small.

A partial converse on Theorem III above is established in Theorem 9.11.

Theorem VII. Let $R$ be local ring. If every homologically finite complex of $R$ modules is virtually small, then $R$ is Gorenstein.

This shows that the class of rings over which every homologically finite complex is virtually small is sandwiched between complete intersections and Gorenstein rings. It is a strict subset of the class of Gorenstein rings as shown by Examples 9.13 and 9.14. If it coincides with the class of complete intersections, then this would give a homotopical characterization in terms of virtual smallness, analogous to the Auslander-Buchsbaum-Serre characterization of regularity in terms of smallness.

A counter-example would be interesting too for it would signal the presence of an interesting - the results in this article testify to this claim - new family of local rings. This aspect of our work is in the same spirit as that of Jorgensen and Şega [21], who have discovered various classes of local rings strictly contained between complete intersections and Gorenstein rings, which nevertheless retain some traces of the homological properties of the former. These classes are not directly related to the one considered here; Examples 9.13 and 9.14 make that clear.

A comment on the exposition: the techniques we use are a mixture of homotopy theory and commutative algebra, and since we hope to interest both algebraists and topologists, we have made a special effort has to provide relevant background when appropriate. 


\section{Complexes}

Let $R$ be a ring; the standing hypothesis in this article is that rings are commutative. Let $M$ be a complex of $R$-modules. Our labeling convention is homological:

$$
M:=\cdots \rightarrow M_{i+1} \stackrel{\partial_{i+1}}{\longrightarrow} M_{i} \stackrel{\partial_{i}}{\longrightarrow} M_{i-1} \rightarrow \cdots .
$$

We set inf $M=\inf \left\{i \mid \mathrm{H}_{i}(M) \neq 0\right\}$ and $\sup M=\sup \left\{i \mid \mathrm{H}_{i}(M) \neq 0\right\}$; in particular, if $\mathrm{H}(M)=0$, then inf $M=\infty$ and $\sup M=-\infty$. When inf $M>-\infty$, respectively, sup $M<\infty$, one says that $\mathrm{H}(M)$ is bounded below, respectively, bounded above. The amplitude of $M$ is the number

$$
\operatorname{amp} M=\sup M-\inf M ;
$$

when this number is finite, then $\mathrm{H}(M)$ is said to be bounded.

If for each integer $i$ the $R$-module $\mathrm{H}_{i}(M)$ is finite, that is to say, finitely generated, then $\mathrm{H}(M)$ is degreewise finite. When $\mathrm{H}(M)$ is both degreewise finite and bounded, we say that $\mathrm{H}(M)$ is finite, or that $M$ is homologically finite. Sometimes, we consider also the property that for each integer $i$, the $R$-module $\mathrm{H}_{i}(M)$ is artinian; in other words, $\mathrm{H}(M)$ is degreewise artinian. When $\mathrm{H}(M)$ is also bounded, we drop the qualifier 'degreewise', or write: $M$ is homologically artinian.

Next, we provide a short resume of the homological algebra of complexes, referring the reader to the work of Avramov and Foxby [3] on homological dimensions for greater detail. Recall that a morphism $\alpha$ of complexes is a quasiisomorphism if $\mathrm{H}(\alpha)$ is bijective.

2.1. Resolutions. A complex $P$ of $R$-modules is projective when it has the property that for each morphism $\alpha: M \rightarrow N$ of complexes, if $\alpha$ is surjective and a quasiisomorphism, the so is

$$
\operatorname{Hom}_{R}(P, \alpha): \operatorname{Hom}_{R}(P, M) \rightarrow \operatorname{Hom}_{R}(P, N) ;
$$

in other words, $P$ preserves surjective quasiisomorphisms. In [3], such a $P$ is said to be DG projective, in order to distinguish it from other flavours of projectives; we omit the 'DG' for this is the only kind of projective complexes considered here.

Each complex $M$ of $R$-modules has a projective resolution: a quasiisomorphism $P \rightarrow M$ with $P$ a projective complex. When $R$ is noetherian and $M$ is homologically finite and bounded below, $P$ may be so constructed that each $P_{i}$ is finitely generated and $P_{i}=0$ for $i<\inf M$. One has also flat complexes and injective complexes, and the corresponding resolutions of $M$.

2.2. Derived functors. The derived category of $R$ is denoted $\mathscr{D}(R)$; when in it the notation $\simeq$ indicates an isomorphism; we recommend Hartshorne [17] and Verdier [29] as basic references on derived categories and derived functors. 
Given complexes $M$ and $N$ of $R$-modules, $M \otimes{ }_{R}^{\mathbf{L}} N$ and $\mathbf{R H o m}_{R}(M, N)$ denote the derived functor of the tensor product functor and the homomorphisms functor, respectively. The existence of projective and injective resolutions ensure that derived functors exist, and that they can be calculated with suitable resolutions of either factor. As usual, for each integer $i$, we set

$$
\operatorname{Tor}_{i}^{R}(M, N)=\mathrm{H}_{i}\left(M \otimes_{R}^{\mathbf{L}} N\right) \quad \text { and } \quad \operatorname{Ext}_{R}^{i}(M, N)=\mathrm{H}_{-i}\left(\operatorname{RHom}_{R}(M, N)\right) .
$$

The existence of resolutions allows one to attach numerical invariants to $M$. One such is the projective dimension of $M$, defined to be the integer

$$
\operatorname{pd}_{R} M=\inf \left\{\begin{array}{l|l}
n \in \mathbb{Z} & \begin{array}{l}
\text { there is projective complex } P \text { with } \\
P \simeq M \text { and } P_{i}=0 \text { for } i \geq n+1
\end{array}
\end{array}\right\} .
$$

Thus, if $\mathrm{H}(M)=0$, then $\operatorname{pd}_{R} M=-\infty$, and if there is no integer $n$ for which an appropriate $P$ exists, then $\operatorname{pd}_{R} M=\infty$. Similarly, $M$ has a flat dimension and an injective dimension, denoted $\mathrm{fd}_{R} M$ and $\operatorname{id}_{R} M$, respectively.

2.3. Minimal resolutions. Let $(R, \mathfrak{m}, k)$ be a local ring, with maximal ideal $\mathfrak{m}$ and residue field $k$. As per the commutative algebraist's convention, 'local' means also noetherian. Each complex $M$ of $R$-modules with $\mathrm{H}(M)$ degreewise finite and bounded below has a minimal free resolution: a quasiisomorphism $F \rightarrow M$ with

$$
F:=\cdots \rightarrow R^{b_{i+1}} \rightarrow R^{b_{i}} \rightarrow R^{b_{i-1}} \rightarrow \cdots
$$

where the $b_{i}$ are integers, and $\partial(F) \subseteq \mathfrak{m} F$; these properties ensure that $b_{i}=0$ for $i<$ $\inf M$. Such a resolution is unique up to isomorphism of complexes. Furthermore, $F$ is a direct summand of any projective resolution of $M$. In particular, $\operatorname{pd}_{R} M \leq n$ if and only if in the minimal free resolution $F$ of $M$ one has $F_{i}=0$ for $i \geq n+1$. Roberts' monograph [26] is an excellent reference for the preceding discussion.

In the sequel, homologically finite complexes of finite projective dimension play an important role, and we use various tests for detecting them. Most of these are available in the literature in a form suitable for our needs; some are not, and so are recorded here. The one below is immediate when we compute the derived functors in question using the minimal resolution of $M$.

2.4. Let $R$ be a local ring and $M$ a complex of $R$-modules which is homologically finite and bounded below. One has equalities

$$
\operatorname{pd}_{R} M=\sup \left(k \otimes_{R}^{\mathbf{L}} M\right)=-\inf \left(\operatorname{RHom}_{R}(M, k)\right) .
$$

When $\operatorname{pd}_{R} M$ is finite, it is also equal to $-\inf \left(\operatorname{RHom}_{R}(M, R)\right)$. 
One application of this test for projective dimension is:

2.5. If $M$ and $N$ are complexes of $R$-modules with $\mathrm{H}(M)$ and $\mathrm{H}(N)$ degreewise finite and bounded below, then

$$
\operatorname{pd}_{R}\left(M \otimes_{R}^{\mathbf{L}} N\right)=\operatorname{pd}_{R} M+\operatorname{pd}_{R} N .
$$

Indeed, this follows from 2.4, since in $\mathscr{D}(R)$ one has isomorphisms

$$
k \otimes_{R}^{\mathbf{L}}\left(M \otimes_{R}^{\mathbf{L}} N\right) \simeq\left(k \otimes_{R}^{\mathbf{L}} M\right) \otimes_{R}^{\mathbf{L}} N \simeq\left(k \otimes_{R}^{\mathbf{L}} M\right) \otimes_{k}^{\mathbf{L}}\left(k \otimes_{R}^{\mathbf{L}} N\right)
$$

where the second one is contained in the remark below, which, in turn, is evident when one uses resolutions of $M$ to realize the derived functors.

2.6. Let $(R, \mathfrak{m}, k)$ be a local ring and $M$ a complex of $R$-modules. Then $k \otimes_{R}^{\mathbf{L}} M$, $\mathbf{R H o m}_{R}(M, k)$, and $\mathbf{R} \operatorname{Hom}_{R}(k, M)$ are isomorphic, in $\mathcal{D}(R)$, to graded $k$-vector spaces with zero differentials.

The next result is a version of 2.4 for non-local rings; it is similar to [3, (5.3.P)].

2.7 Proposition. Let $R$ be a noetherian ring and $M$ a complex of $R$-modules with $\mathrm{H}(M)$ degreewise finite and bounded below. If the following conditions hold:

(a) $\operatorname{RHom}_{R}(M, R / \mathfrak{m})$ is homologically bounded below for each maximal ideal $\mathfrak{m}$ of $R$,

(b) $\operatorname{RHom}_{R}(M, R)$ is homologically bounded below,

then $\operatorname{pd}_{R} M=-\inf \mathbf{R H o m}_{R}(M, R)$, and in particular, $\operatorname{pd}_{R} M<\infty$.

Proof. Set $d=-\inf \mathbf{R H o m}_{R}(M, R)$; possibly, $d=-\infty$, which happens when $\mathbf{R H o m}_{R}(M, R) \simeq 0$. Evidently, $\operatorname{pd}_{R} M \geq d$. For the reverse inequality, it is enough to check that $\mathrm{pd}_{R_{\mathfrak{p}}} M_{\mathfrak{p}} \leq d$ for each prime ideal $\mathfrak{p}$ of $R$; see [3, (5.3.P)]. Since projective dimension is non-increasing upon localization, it suffices to verify this last inequality at each maximal ideal of $R$.

Fix a maximal ideal $\mathfrak{m}$ of $R$ and let $k$ be the residue field $R / \mathfrak{m}$. Since $R$ is noetherian and $\mathrm{H}(M)$ is degreewise finite and bounded below, one obtains

$$
\begin{array}{ll}
\operatorname{Ext}_{R_{\mathfrak{m}}}^{i}\left(M_{\mathfrak{m}}, k\right) \cong \operatorname{Ext}_{R}^{i}(M, R / \mathfrak{m})_{\mathfrak{m}}=0 & \text { for all } i \gg 0, \\
\operatorname{Ext}_{R_{\mathfrak{m}}}^{i}\left(M_{\mathfrak{m}}, R_{\mathfrak{m}}\right) \cong \operatorname{Ext}_{R}^{i}(M, R)_{\mathfrak{m}}=0 & \text { for all } i \geq d+1 .
\end{array}
$$

The complex $M_{\mathfrak{m}}$ of $R_{\mathfrak{m}}$-modules is homologically degreewise finite and bounded below, so the vanishing in the first row implies that $\operatorname{pd}_{R_{\mathfrak{m}}} M_{\mathfrak{m}}$ is finite, by 2.4. Given this, the vanishing in the second row yields $\operatorname{pd}_{R_{\mathfrak{m}}} M_{\mathfrak{m}} \leq d$, again by 2.4 . This is the desired conclusion. 
2.8. Koszul complexes. Let $\boldsymbol{x}$ be a finite set of elements in $R$. For each complex $M$ of $R$-modules, the Koszul complex on $\boldsymbol{x}$ with coefficients in $M$ is denoted $\mathrm{K}[\boldsymbol{x} ; M]$. One way to construct it is by using the recipe: $\mathrm{K}[\emptyset ; M]=M$, and for $\boldsymbol{y}=\boldsymbol{x} \sqcup\{y\}$ the complex $\mathrm{K}[\boldsymbol{y} ; M]$ is the mapping cone of the endomorphism

$$
\mathrm{K}[\boldsymbol{x} ; M] \rightarrow \mathrm{K}[\boldsymbol{x} ; M] \quad \text { where } m \mapsto y m \text { for } m \in \mathrm{K}[\boldsymbol{x} ; M] .
$$

The reader may consult Bruns and Herzog $[8, \S(1.6)]$ for details.

Let $(R, \mathfrak{m}, k)$ be a local ring and $\boldsymbol{x}$ a minimal generating set for $\mathfrak{m}$; Nakayama's lemma implies $\operatorname{card}(\boldsymbol{x})=\operatorname{rank}_{k}\left(\mathfrak{m} / \mathfrak{m}^{2}\right)$. One refers to $\mathrm{K}[\boldsymbol{x} ; R]$ as the Koszul complex of $R$. No reference is made to $\boldsymbol{x}$ because if $\boldsymbol{y}$ is another minimal generating set for $\mathfrak{m}$, then $\mathrm{K}[\boldsymbol{x} ; R] \cong \mathrm{K}[\boldsymbol{y} ; R]$ as complexes of $R$-modules; see the discussion after $[8,(1.6 .20)]$.

The following properties of the Koszul complex are well known.

2.9. Let $R$ be a ring, $M$ a complex of $R$-modules, and $\boldsymbol{x} \subset R$ a finite set.

(a) The ideal (x) annihilates $\mathrm{H}(\mathrm{K}[\boldsymbol{x} ; M])$.

(b) If $\mathrm{H}(M)$ is degreewise noetherian, then so is $\mathrm{H}(\mathrm{K}[x ; M])$.

(c) If $\mathrm{H}(M)$ is degreewise artinian, then so is $\mathrm{H}(\mathrm{K}[x ; M])$.

(d) If $\operatorname{amp}(M)$ is finite, then so is $\operatorname{amp}(\mathrm{K}[\boldsymbol{x} ; M])$; the converse holds when $\boldsymbol{x}$ is in the Jacobson radical of $R$ and the $R$-module $\mathrm{H}(M)$ is degreewise noetherian.

Indeed, (b) and (c) are immediate from the description of $\mathrm{K}[\boldsymbol{x} ; M]$ as an iterated mapping cone, as is the first claim in (d); for its converse, one uses Nakayama's lemma. For (a), one constructs an explicit homotopy between multiplication by $x$ and by zero on $\mathrm{K}[\boldsymbol{x} ; M]$; see $[8,(1.6 .5)]$.

\section{Thick subcategories of derived categories}

Let $R$ be a ring; recall that $\mathscr{D}(R)$ denotes the derived category of $R$-modules. This work makes critical use of the triangulated structure on $\mathscr{D}(R)$; once again, [17] and [29] are suitable references for this topic. The aim of this section is to recall certain important notions based on the triangulated structure, and to provide numerous examples to illustrate the ideas involved.

3.1. A thick subcategory $\mathcal{T}$ of $\mathscr{D}(R)$ is a non-empty full subcategory such that:

(a) $\mathcal{T}$ is closed under isomorphisms in $\mathscr{D}(R)$.

(b) In any exact triangle, if two of the objects are in $\mathcal{T}$, then so is the third. 
(c) Every direct summand of an object in $\mathcal{T}$ is also in $\mathcal{T}$.

Thick subcategories are synonymous with the épaisse subcategories of Verdier [29], although the definition in loc. cit. differs from the one given here; the formulation above is due to Rickard [27]. If a thick subcategory $\mathcal{T}$ is closed under homotopy colimits, then it is said to be localizing; see [24].

3.2 Example. The following subcategories of $\mathscr{D}(R)$ are thick:

(i) The subcategory of complexes of finite flat dimension.

(ii) The subcategory of complexes of finite projective dimension.

(iii) The subcategory of complexes of finite injective dimension.

When $R$ is noetherian, the following subcategories are also thick:

(iv) The subcategory of complexes whose homology is (degreewise) finite.

(v) The subcategory of complexes whose homology is (degreewise) artinian.

We verify that the subcategory defined in (i), which we denote $\mathcal{F}$, satisfies the conditions in 3.1, and hence thick; the arguments for the other subcategories listed above are similar. The simplest method of checking that $\mathcal{F}$ is thick is to take recourse to a characterization of finite flat dimension in terms of derived functors: a complex $F$ of $R$-modules has flat dimension $\leq n$ if and only if $\sup \left(F \otimes_{R}^{\mathbf{L}} X\right) \leq n$ for each $R$-module $X$; see [3, (2.4.F)].

Clearly, $\mathcal{F}$ is closed under isomorphisms; this takes care of (a). As to (b), let

$$
L \longrightarrow M \longrightarrow N \longrightarrow \Sigma L
$$

be an exact triangle in $\mathscr{D}(R)$. For each $R$-module $X$, the homology long exact sequence arising from the exact triangle

$$
\left(L \otimes_{R}^{\mathbf{L}} X\right) \longrightarrow\left(M \otimes_{R}^{\mathbf{L}} X\right) \longrightarrow\left(N \otimes_{R}^{\mathbf{L}} X\right) \longrightarrow \Sigma\left(L \otimes_{R}^{\mathbf{L}} X\right)
$$

implies that if any two of the numbers $\sup \left(L \otimes_{R}^{\mathbf{L}} X\right), \sup \left(M \otimes_{R}^{\mathbf{L}} X\right)$, and $\sup \left(N \otimes_{R}^{\mathbf{L}} X\right)$, is $\leq n$, then the third is $\leq n+1$. Thus, if any two of $L, M$, and $N$ are in $\mathcal{F}$, then so is the third; this settles (b). Finally, if $N$ is a direct summand of $M$, then $N \otimes_{R}^{\mathbf{L}} X$ is a direct summand of $M \otimes_{R}^{\mathbf{L}} X$, so $\sup \left(N \otimes_{R}^{\mathbf{L}} X\right) \leq \sup \left(M \otimes_{R}^{\mathbf{L}} X\right)$. Thus, $M$ in $\mathcal{F}$ implies $N$ in $\mathcal{F}$, as desired.

Note that none of the subcategories (i)-(v) is localizing, for they are not closed under homotopy colimits. Here is an example of a localizing subcategory; see, for example, $[12,(5.3)]$ for a proof.

3.3 Example. Let $I$ be an ideal in noetherian ring $R$. The subcategory of $\mathscr{D}(R)$ consisting of complexes $M$ where $\mathrm{H}_{i}(M)$ is $I$-torsion for each integer $i$, is localizing. 
3.4. Let $M$ be a complex of $R$-modules. The intersection of thick subcategories of $\mathscr{D}(R)$ containing $M$ is itself a thick subcategory. We refer to it as the thick subcategory generated by $M$, and denote it Thick ${ }_{R}[M]$. It is suggestive to think of complexes in Thick $_{R}[M]$ as being finitely built from $M$.

In the same vein, there is a smallest (with respect to inclusion) localizing subcategory generated by $M$, and we speak of any object in this subcategory as being built from $M$. This subcategory does not arise often in this work, so we resist the temptation to introduce notation for it.

3.5 Example. Let $(R, \mathfrak{m}, k)$ be a local ring. A complex $M$ of $R$-modules is built from $k$ if and only if for each integer $i$, the $R$-module $\mathrm{H}_{i}(M)$ is $\mathfrak{m}$-torsion; it is finitely built from $k$ if and only the length of the $R$-module $\mathrm{H}(M)$ is finite.

Indeed, the criterion for $M$ being built from $k$ is a special case of the preceding example, and once again we refer the reader to [12] for details. As to the one for being finitely built, it is not hard to verify that the subcategory of complexes with finite length homology is thick; since this subcategory contains $k$, it must contain also $\operatorname{Thick}_{R}[k]$. This means that anything finitely built from $k$ has homology of finite length. Conversely, let $M$ be a complex such that the length of $\mathrm{H}(M)$ is finite; thus, $\mathrm{H}(M)$ is bounded and the length of each $\mathrm{H}_{i}(M)$ is finite. Since $R$ is local, the only simple module is $k$, so $\mathrm{H}_{i}(M)$ admits a finite filtration with subquotients isomorphic to $k$; this implies that, in $\mathscr{D}(R)$, each $\mathrm{H}_{i}(M)$ is finitely built from $k$. Now an straightforward induction on the amplitude of $M$ yields that $M$ is finitely built from $k$; see 3.10 .

The localizing subcategory generated by $R$ is all of $\mathscr{D}(R)$; this is another way of saying that each complex is isomorphic to one consisting of projective modules. The thick subcategory generated by $R$ plays a prominent role in our investigations, so it is convenient to introduce some language to address complexes in it.

3.6. Small complexes. We say that a complex of $R$-modules is small if it is finitely built from $R$. The following result reconciles the definition adopted here with those discussed in the introduction. Our choice of definition was dictated by closeness to the spirit of this paper.

3.7. Let $M$ be a complex of $R$-modules. The following conditions are equivalent:

(a) $M$ is small;

(b) $M$ is isomorphic in $\mathscr{D}(R)$ to a finite complex of finite projective modules;

(c) $\operatorname{RHom}_{R}(M,-)$ commutes with arbitrary direct sums in $\mathscr{D}(R)$.

This result is well known; the details are given for the sake of readability. Also, we sketch only the proof that (a) and (b) are equivalent, for that is all we use in this article. 
(a) $\Longleftrightarrow$ (b): It is not hard to verify that the class of complexes isomorphic in $\mathscr{D}(R)$ to finite complexes of finite projectives is thick, and since it contains $R$, it contains also Thick ${ }_{R}[R]$, that is to say, every small complex. For the converse, it suffices to prove each complex of the form

$$
P:=0 \rightarrow P_{n} \rightarrow \cdots \rightarrow P_{m} \rightarrow 0
$$

where each $R$-module $P_{i}$ is finite and projective, is in $\operatorname{Thick}_{R}[R]$. To this end we induce on $n-m$. First note that Property (b) in 3.1 implies that any finite free module is in $\operatorname{Thick}_{R}[R]$, and hence by Property (c) of loc. cit., so is any direct summand of such a module. Thus, any finite projective module is in $\operatorname{Thick}_{R}[R]$, which settles the base case $n=m$. As to the induction step, assuming $n-m \geq 1$, note that $P$ is the mapping cone of the morphism of complexes $\partial_{n}^{P}: \Sigma^{n-1} P_{n} \rightarrow P_{\leqslant n-1}$, so in $\mathscr{D}(R)$ one has a distinguished triangle

$$
P_{\leqslant n-1} \longrightarrow P \longrightarrow \Sigma^{n-1} P_{n} \longrightarrow \Sigma P_{\leqslant n-1} .
$$

Now, the induction hypothesis yields that $P_{\leqslant n-1}$ and $\Sigma^{n-1} P_{n}$ are in $\operatorname{Thick}_{R}[R]$, and hence so is $P$, by Property (b) of 3.1 .

Over a noetherian ring, a homologically finite complex is isomorphic in the derived category to a finite complex of finite projectives if and only if it has finite projective dimension, if and only if it has finite flat dimension. Thus, over such rings, the equivalence (a) $\Longleftrightarrow$ (b) of the preceding result yields the following homological characterization of smallness.

3.8. Let $R$ be a noetherian ring and $M$ a homologically finite complex of $R$-modules. The following conditions are equivalent:

(a) $M$ is small;

(b) $\operatorname{pd}_{R} M$ is finite;

(c) $\mathrm{fd}_{R} M$ is finite.

We record some elementary remarks concerning the process of building one thing out of another. In presenting these, it is expedient to introduce the following notation: given complexes $M$ and $N$ of $R$-modules, we write

$$
M \underset{R}{\Longrightarrow} N
$$

to indicate that $N$ is in $\operatorname{Thick}_{R}[M]$, that is to say, that $N$ is finitely built from $M$. Our reason for adopting this symbolism is that, often, properties of a complex $M$ are inherited by complexes finitely built from it. We hope that the discussion to follow will convince the reader that the language, of one complex being built from another, and the notation, $M \Longrightarrow N$, aid intuition about these notions.

In the following remarks, $L, M$, and $N$ are complexes of $R$-modules. 
3.9. If $M$ is finitely built from $L$, and $N$ is finitely built from $M$, then $N$ is finitely built from L. In pictures:

$$
L \underset{R}{\Longrightarrow} M \text { and } M \underset{R}{\Longrightarrow} N \text { entails } L \underset{R}{\Longrightarrow} N
$$

The assertion above is verified by a direct appeal to the definition of $\operatorname{Thick}_{R}[-]$.

3.10. If $\mathrm{H}(N)$ is bounded and for each integer $i$ the $R$-module $\mathrm{H}_{i}(N)$ is finitely built from $M$, then $N$ is finitely built from $M$.

Indeed, this claim is evidently true when $\operatorname{amp} N=0$, for then $N \simeq \mathrm{H}_{i}(N)$ for some $i \in \mathbb{Z}$. This is the basis of an induction on $\operatorname{amp} N$. As to the induction step: for $s=\sup N$, by suitably truncating $N$ one can construct an exact triangle

$$
\Sigma^{s} \mathrm{H}_{s}(N) \rightarrow N \rightarrow \tilde{N} \rightarrow \Sigma^{s+1} \mathrm{H}_{s}(N)
$$

where the induced map $\mathrm{H}_{i}(N) \rightarrow \mathrm{H}_{i}(\tilde{N})$ is bijective for $i \neq s$, and $\mathrm{H}_{s}(\tilde{N})=0$. Therefore, the induction hypothesis yields that Thick ${ }_{R}[M]$ contains both $\Sigma^{s} \mathrm{H}_{s}(N)$, because its amplitude is zero, and $\widetilde{N}$, because its amplitude is strictly less than the amplitude of $N$. Since Thick ${ }_{R}[M]$ is closed under exact triangles, one concludes that it contains $N$ as well.

Caveat: the converse to 3.10 need not hold, as the next example demonstrates.

3.11 Example. Let $(R, \mathfrak{m}, k)$ be a local ring and $K$ the Koszul complex of $R$. Given 3.8 , it is clear that $K$ is finitely built from $R$, while $\mathrm{H}_{0}(K)$, that is to say, $k$, is finitely built from $R$ if and only if the ring $R$ is regular.

3.12. Let $\varphi: R \rightarrow S$ be a homomorphism of rings, and $P$ a complex of $S$-modules.

$$
M \underset{R}{\Longrightarrow} N \text { entails }\left\{\begin{array}{c}
\left(P \otimes_{R}^{\mathbf{L}} M\right) \underset{S}{\Longrightarrow}\left(P \otimes_{R}^{\mathbf{L}} N\right), \\
\mathbf{R}_{R}(P, M) \stackrel{\Longrightarrow}{\Longrightarrow} \mathbf{R H o m}_{R}(P, N), \\
\operatorname{RHom}_{R}(M, P) \underset{S}{\Longrightarrow} \mathbf{R H o m}_{R}(N, P) .
\end{array}\right.
$$

The claims above are immediate, for the functors $P \otimes_{R}^{\mathbf{L}}-, \mathbf{R H o m}_{R}(P,-)$, and $\mathbf{R H o m}_{R}(-, P)$ from $\mathscr{D}(R)$ to $\mathscr{D}(S)$ respect the triangulated structures.

For the next remark, which is also easy to verify, recall that given a homomorphism $\psi: Q \rightarrow R$ of rings, one may view $M$ and $N$ as complexes of $Q$-modules, by restriction along $\psi$. 
3.13. Let $\psi: Q \rightarrow R$ be a homomorphism of rings. If, as complexes of $R$-modules, $N$ is finitely built from $M$, then the same is true when they are viewed as complexes of $Q$-modules. In pictures:

$$
M \underset{R}{\Longrightarrow} N \text { implies } M \underset{Q}{\Longrightarrow} N .
$$

Note that the converse of the preceding remark does not hold.

3.14 Example. Let $Q$ be a ring, $\psi$ the canonical inclusion $Q \rightarrow Q[x] /\left(x^{2}\right)=R$, and let $N$ be the $R$-module $R /(x) \cong Q$. As a $Q$-module, $R \cong N \oplus N$, so $N$ is finitely built from $R$. However, it is easy to prove that as $R$-modules $N$ is not finitely built from $R$.

Next, we recall a notion of support for complexes, introduced by Foxby [14].

3.15. Support. Let $R$ be a ring. Given a prime ideal $\mathfrak{p}$, we denote $\boldsymbol{k}(\mathfrak{p})$ its residue class field $R_{\mathfrak{p}} / \mathfrak{p} R_{\mathfrak{p}}$ at $\mathfrak{p}$. The small support of a complex $M$ of $R$-modules is the subset of $\operatorname{Spec} R$ described by

$$
\operatorname{supp}_{R} M=\left\{\mathfrak{p} \in \operatorname{Spec} R \mid\left(\boldsymbol{k}(\mathfrak{p}) \otimes_{R}^{\mathbf{L}} M\right) \not \varkappa 0\right\} .
$$

Usually, we drop the subscript ' $R$ ' from $\operatorname{supp}_{R} M$, unless there is cause for confusion.

The small support of $M$ is a subset of the support of $M$, which is the set

$$
\operatorname{Supp}_{R} M=\left\{\mathfrak{p} \in \operatorname{Spec} R \mid M_{\mathfrak{p}} \nsucceq 0\right\},
$$

whence the name 'small support'. This inclusion can be strict; for example, the small support of $\boldsymbol{k}(\mathfrak{p})$ is $\{\mathfrak{p}\}$, while its support is $\mathrm{V}(\mathfrak{p})$. Recall that for any ideal $I$ in $R$, the set $\{\mathfrak{q} \in \operatorname{Spec} R \mid \mathfrak{q} \supseteq I\}$ is denoted $\mathrm{V}(I)$.

For homologically finite complexes over noetherian rings, the two notions coincide. This is the gist of Part (d) of the remark below, which lists also other basic properties of the small support.

3.16. Let $R$ be a ring and let $M$ and $N$ be complexes of $R$-modules.

(a) For each finite subset $\boldsymbol{x}$ of $R$ one has $\operatorname{supp}_{R} \mathrm{~K}[\boldsymbol{x} ; R]=\mathrm{V}(\boldsymbol{x})$.

(b) If $N$ is built from $M$, then $\operatorname{supp}_{R} N \subseteq \operatorname{supp}_{R} M$.

(c) $\operatorname{supp}_{R}\left(M \otimes \otimes_{R}^{\mathbf{L}} N\right)=\operatorname{supp}_{R} M \cap \operatorname{supp}_{R} N$.

(d) When $R$ is noetherian and $\mathrm{H}(M)$ finite, one has equalities

$$
\operatorname{supp}_{R} M=\bigcup_{i \in \mathbb{Z}} \operatorname{Supp}_{R} \mathrm{H}_{i}(M)=\operatorname{Supp}_{R} M .
$$

In particular, $\operatorname{supp}_{R} M$ is a closed subset of $\operatorname{Spec} R$. 
Indeed, (a) is a routine verification, as is (c), as long as we keep 2.6 in mind. For (d), see the discussion preceding [14, (2.6)], while the key to the proof of (b) is that (derived) tensor products commute with homotopy colimits.

We end this section by recalling the following remarkable theorem discovered by M. Hopkins [18, (11)]; also see Neeman [24, (1.2), (2.8)] and Thomason [28, (3.15)]. It is a powerful test for detecting when one complex is (finitely) built from another.

3.17. Let $R$ be a noetherian ring and let $M$ and $N$ be complexes of $R$-modules. If $\operatorname{supp} N \subseteq \operatorname{supp} M$, then $N$ is built from $M$; it is also finitely built from $M$ when both $M$ and $N$ are small.

\section{Virtually small complexes}

Let $R$ be a commutative ring. This section introduces two new classes of complexes, which are the focus of study in this article, and develops some of the elementary theory concerning them.

4.1. Virtually small complexes. A complex $M$ of $R$-modules, with $\mathrm{H}(M) \neq 0$, is virtually small if Thick $R_{R}[M]$ contains a non-zero small complex; in other words, there is a complex $W$ of $R$-modules, with $\mathrm{H}(W) \neq 0$, such that

(i) $W$ is finitely built from $R$, and

(ii) $W$ is finitely built from $M$.

The complex $M$ is proxy small if in addition to (i) and (ii) one has that

(iii) $M$ is built from $W$

By decree, a homologically zero complex is proxy small, and hence also virtually small. The complex $W$ is said to be a witness that $M$ is virtually small, or a witness that $M$ is proxy small, as is appropriate. When the property in question is clear from the context, we state simply that $W$ is a witness for $M$.

Proxy small complexes were introduced in [13], where they played a crucial role in the development of a framework for studying duality phenomenon in algebra and topology. From the perspective of homological algebra, the notion of 'virtual smallness' is a natural generalization of that of 'smallness', for the behaviour of virtually small complexes is similar to that of small complexes; this is borne out by the results established in this article. First, the obvious example.

4.2. Each small complex is proxy small, and hence also virtually small. 
In particular, when the ring $R$ is regular, every homologically finite complex is proxy small. However, there are many more; confer Corollary 4.10 and Proposition 4.11. Even over an arbitrary ring there is a plethora of proxy small complexes.

4.3 Example. Let $X$ be complex of $R$-modules and set $M=R \oplus X$. Since $R$ is a direct summand of $M$, it is contained in Thick ${ }_{R}[M]$. Hence $M$ is virtually small; it is even proxy small because $M$ is built from $R$. Note that $M$ is small if and only if $X$ is small.

One of the difficulties in checking whether a complex is virtually small or proxy small is that there are no canonical witnesses. However, the situation is under better control over noetherian rings; this is the content of the next two propositions.

4.4 Proposition. Let $R$ be a noetherian ring and $M$ a complex of $R$-modules. The following conditions are equivalent.

(a) The complex $M$ is proxy small.

(b) The subset $\operatorname{supp} M \subseteq \operatorname{Spec} R$ is closed, and each small complex $P$ with supp $P \subseteq \operatorname{supp} M$ is finitely built from $M$.

(c) There is a finite subset $\boldsymbol{x}$ of $R$ with $\mathrm{V}(\boldsymbol{x})=\operatorname{supp} M$ and $\mathrm{K}[\boldsymbol{x} ; R]$ finitely built from $M$.

Proof. (a) $\Longrightarrow$ (b): Let $W$ be a witness that $M$ is proxy small. Thus, $W$ and $M$ are built from each other, so $\operatorname{supp} W=\operatorname{supp} M$, by 3.16 (b). But then, $W$ is homologically finite so 3.16 (d) yields that supp $W$ is a closed subset of Spec $R$, and hence the same is true of $\operatorname{supp} M$. Since the complexes $W$ and $P$ are small and supp $P \subseteq \operatorname{supp} M$, one obtains from 3.17 that $P$ is finitely built from $W$. Therefore, 3.9 implies that $P$ is finitely built also from $M$, as claimed.

(b) $\Longrightarrow$ (c): Take $\boldsymbol{x}$ to be a finite set with $\mathrm{V}(\boldsymbol{x})=\operatorname{supp} M$.

(c) $\Longrightarrow$ (a): Given that $\mathrm{K}[\boldsymbol{x} ; R]$ is small it suffices to verify that $M$ is built from it. By 3.16 (a), the support of $\mathrm{K}[\boldsymbol{x} ; R]$ is $\mathrm{V}(\boldsymbol{x})$, which equals supp $M$, so 3.17 yields the desired conclusion.

There is a recognition principle also for virtual smallness; it is especially useful over local rings.

4.5 Proposition. Let $R$ be a noetherian ring and $M$ a complex of $R$-modules with $\mathrm{H}(M) \neq 0$. The following conditions are equivalent.

(a) The complex $M$ is virtually small.

(b) The subset $\operatorname{supp} M \subseteq \operatorname{Spec} R$ contains a closed point $\{\mathfrak{m}\}$ with the property that each small complex supported on $\{\mathfrak{m}\}$ is finitely built from $M$. 
(c) There is a finite set $\boldsymbol{x} \subset R$ such that $(\boldsymbol{x}) \neq R$ and $\mathrm{K}[\boldsymbol{x} ; R]$ is finitely built from $M$.

In particular, when $R$ is local, $M$ is virtually small if and only if the Koszul complex of $R$ is finitely built from $M$.

Proof. (a) $\Longrightarrow$ (b): For each witness $W$ that $M$ is virtually small, the subset supp $W \subset \operatorname{Spec} R$ is non-empty and closed; this follows from 3.16(d). Let $\{\mathfrak{m}\}$ be a closed point in supp $W$. If $P$ is a small complex with supp $P=\{\mathfrak{m}\}$, it follows from 3.17 that $P$ is finitely built from $W$, and hence that it is finitely built from $M$, by 3.9.

For (b) $\Longrightarrow$ (c) set $\boldsymbol{x}$ to be a finite generating set for the ideal $\mathfrak{m}$, while (c) $\Longrightarrow$ (a) is almost by definition; all one needs to note is that supp $\mathrm{K}[\boldsymbol{x} ; M]$ is non-empty, by $3.16(a)$.

4.6 Remark. The preceding propositions are based on Hopkins' theorem 3.17, and it is for this reason that the bulk of the results presented in this article are restricted to commutative noetherian rings. There are extensions of the Hopkins' theorem to other categories: modules over commutative, but not necessarily noetherian, rings, sheaves on a scheme, and modules over group rings. In all these the role of thick subcategories is played by the less restrictive class of tensor closed thick subcategories, which suggests that the 'correct' definition of virtual smallness and proxy smallness should involve such subcategories.

The examples given so far of proxy small, or virtually small, complexes are based on small complexes. Next we identify diverse classes of virtually small complexes; these demonstrate that there is a lot more to these notions than smallness.

The result below will be subsumed in Proposition 7.6. However, it seemed worthwhile to give 'ad hoc' arguments for this one, for the strategy employed is useful also in other cases.

4.7 Proposition. For each local ring $(R, \mathfrak{m}, k)$, the $R$-module $k$ is proxy small.

Proof. We verify that the Koszul complex $K$ of $R$ is the witness we seek, as predicted by Proposition 4.4. In 4.1, condition (i) asks that $K$ be small, and that we know, while (ii) is a consequence of 3.10: since $k$ is a field and $\mathrm{H}(K)$ is a finite dimensional $k$ vector space, $\mathrm{H}(K)$ is finitely built from $k$ in $\mathscr{D}(k)$, and hence also in $\mathscr{D}(R)$, by 3.13. One way to check (iii) is to invoke 3.17, as supp $k=\operatorname{supp} K$. Another is to note that $K$ is a Differential Graded $R$-algebra and $k$ a DG $K$-module, via the augmentation $K \rightarrow k$. Thus, $k$ is built from $K$ as a DG $K$-module, and hence also as an $R$-module, by a DG analogue of 3.13 .

The proposition above takes on greater significance in the light of the following example that shows that not everything is virtually small. We defer our justification 
to 5.8, for it is based on certain general results on virtually small complexes; we do not know how to verify this directly.

4.8 Example. Set $R=k[x, y] /\left(x^{2}, x y, y^{2}\right)$. The $R$-module $R /(y)$ is not virtually small.

One fall-out of this example and the proposition above is that $k$ cannot be used as a 'test' object for detecting rings over which every homologically finite complex is virtually small.

Over a local ring, if a complex of finite flat dimension is homologically finite, then it is small - see 3.8 - and hence virtually small. The following result extends this remark, and illustrates the flexibility afforded by the notion of virtual smallness. Its proof is rather long and involved; as is explained in it, the difficulty is in dealing with the case when the field extension $k \rightarrow l$ is infinite. One comment: not every flat module is virtually small; see Example 4.17.

4.9 Theorem. Let $\varphi:(R, \mathfrak{m}, k) \rightarrow(S, \mathfrak{n}, l)$ be a local homomorphism and $N$ a complex of $S$-modules with $\mathrm{H}(N)$ either finite or artinian. If $\operatorname{fd}_{R} N$ is finite, then $N$ is virtually small over $R$.

Proof. We may assume that $\mathrm{H}(N)$ is an $l$-vector space of finite rank. Indeed, let $K$ be the Koszul complex on $S$. By 3.12, since $K$ is finitely built from $S$, the complex $K \otimes_{S} N$ is finitely built from $N$. This holds in $\mathscr{D}(S)$, and hence also in $\mathscr{D}(R)$, by 3.13. Consequently, it is enough to prove that $K \otimes_{S} N$ is virtually small over $R$. Moreover, $\mathrm{fd}_{R}\left(K \otimes_{S} N\right)$ is finite; one way to see this is use 3.2 (i), since $K \otimes_{S} N$ is finitely built from $N$. The $S$-module $\mathrm{H}\left(K \otimes_{S} N\right)$ is either finite or artinian, because $\mathrm{H}(N)$ has this property, and annihilated by $\mathfrak{n}$; both these deductions use 2.9. Thus, replacing $N$ with $K \otimes_{S} N$ accomplishes the desired reduction.

At this point, in the special case where $l$ has finite rank over $k$, one obtains that $\mathrm{H}(N)$ is finite as a $k$-vector space, and hence also as an $R$-module. Therefore, the finiteness of $\operatorname{fd}_{R} N$ yields that the complex of $R$-modules $N$ is small, by 3.8, and hence also virtually small.

When the extension of residue fields is not finite the argument is a lot more involved, and is a classic devissage argument. First, we reduce to the case where both $R$ and $S$ are complete as follows: let $\widehat{R}$ denote the $\mathfrak{m}$-adic completion of $R$, and let $\widehat{S}$ denote the $\mathfrak{n}$-adic completion of $S$. We claim that when $\widehat{S} \otimes_{S} N$ is virtually small over $\widehat{R}$, the complex $N$ is virtually small over $R$. To see this, let $K$ be the Koszul complex of $R$. Thus $\widehat{R} \otimes_{R} K$ is the Koszul complex of $\widehat{R}$, so when $\widehat{S} \otimes_{R} N$ is virtually small over $\widehat{R}$, one has that

$$
N \underset{S}{\stackrel{\simeq}{\longrightarrow}} \widehat{S} \otimes_{S} N \underset{\widehat{R}}{\longrightarrow} \widehat{R} \otimes_{R} K \underset{R}{\stackrel{\simeq}{R}} K .
$$


In this diagram, the arrow pointing right is a quasiisomorphism because $S \rightarrow \widehat{S}$ is flat and $\mathfrak{n} \mathrm{H}(N)=0$, while the arrow pointing left is a quasiisomorphism because $R \rightarrow \widehat{R}$ is flat and $\mathfrak{m} \mathrm{H}(K)=0$. The implication is given by Proposition 4.5. In particular, using 3.13, one obtains that $K$ is finitely built from $N$, that is to say, $N$ is virtually small over $R$. To complete the reduction to the complete case, one has to verify that the flat dimension of $\widehat{S} \otimes_{S} N$ over $\widehat{R}$ is finite. Now, since $\mathrm{fd}_{R} N$ is finite, base change yields that $\mathrm{fd}_{\widehat{R}}\left(\widehat{R} \otimes_{R} N\right)$ is finite. On the other hand, the quasiisomorphism $N \rightarrow \widehat{S} \otimes_{S} N$ factors as

$$
N \rightarrow \widehat{R} \otimes_{R} N \rightarrow \widehat{S} \otimes_{S} N .
$$

Since $\mathfrak{m} \mathrm{H}(N)=0$ the first map in the diagram above is a quasiisomorphism and hence so is the second. Thus, $\operatorname{fd}_{\widehat{R}}\left(\widehat{S} \otimes_{S} N\right)$ is finite, as desired.

Summing up, it remains to prove: given a local homomorphism $\varphi: R \rightarrow(S, \mathfrak{n}, l)$ of complete local rings and a homologically finite complex $N$ of $S$-modules, if $\operatorname{fd}_{R} N$ is finite, then $N$ is virtually small over $R$. We no longer assume that $\mathfrak{n} \cdot \mathrm{H}(N)=0$, for that plays no role in the sequel.

The next step is to construct a convenient factorization of $\varphi$, and reduce the problem to one about complexes over regular local rings. The local ring $R$ being complete, Cohen's structure theorem gives a surjective map $\pi:(P, \mathfrak{p}, k) \rightarrow R$ with $P$ a regular local ring. We embed this into a commutative diagram of local homomorphisms

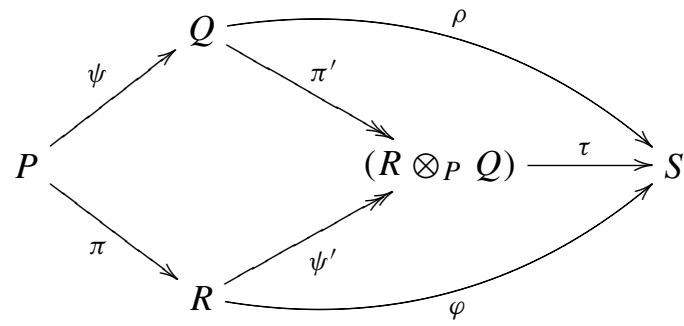

with the following properties:

(a) $Q$ is a regular local ring and $\rho$ is a surjective homomorphism;

(b) $\psi$ is a flat local homomorphism whose fibre $Q / \mathfrak{p} Q$ is regular;

(c) $\psi^{\prime}=\left(R \otimes_{P} \psi\right), \pi^{\prime}=\left(\pi \otimes_{P} Q\right)$, and $\tau(r \otimes q)=\varphi(r) \rho(q)$.

This diagram is constructed as follows: since $S$ is complete applying the Cohen factorization theorem of Avramov, Foxby, and B. Herzog [5, (1.1)] to $\varphi \circ \pi$ provides the surjective homomorphism $\rho$, and the flat local homomorphism $\psi$ with regular fibre. Since $P$ is regular, the last two properties entail $Q$ regular; see [8, (2.2.12)]. Property (c) prescribes the remaining homomorphisms in the diagram.

Now we return to the complex of $S$-modules $N$, which you will recall is homologically finite with $\mathrm{fd}_{R} N$ finite; we may also assume that $\mathrm{H}(N) \neq 0$. The desired 
conclusion is that $N$ is virtually small over $R$, and for this it suffices to prove: the Koszul complex $K$ of $Q$ is virtually small over $P$.

Indeed, we claim more precisely that if $W$ is a witness that $K$ is virtually small over $P$, then the complex of $R$-modules $R \otimes_{P} W$ is a witness that $N$ is virtually small over $R$. By construction the homomorphism $\tau$ is surjective; in particular, $\mathrm{H}(N)$ is finite also over the local ring $R^{\prime}=R \otimes_{P} Q$. The homomorphism $\psi^{\prime}$ is obtained by base change of the flat homomorphism $\psi$ along $\pi$, so it is also flat, and its fibre is isomorphic to that of $\psi$, and hence a regular local ring. Thus, $\tau \circ \psi^{\prime}$ is a Cohen

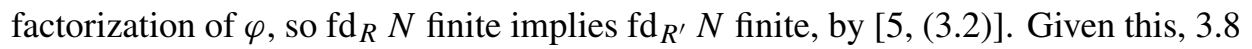
yields that when viewed as a complex of $R^{\prime}$-modules $N$ is small. Now, the complex $R^{\prime} \otimes_{Q} K$ is small, by base change, and its support is the maximal ideal of $R^{\prime}$, by 3.16 (a). Since $\operatorname{supp}_{R^{\prime}}\left(R^{\prime} \otimes_{Q} K\right) \subseteq \operatorname{supp}_{R^{\prime}} N$, and both complexes in question are small, from 3.17 one obtains the first of the following implications

$$
N \underset{R^{\prime}}{\Longrightarrow}\left(R^{\prime} \otimes_{Q} K\right)=\left(R \otimes_{P} Q\right) \otimes_{Q} K \cong\left(R \otimes_{P} K\right) \underset{R}{\Longrightarrow}\left(R \otimes_{P} W\right) .
$$

The second implication holds by 3.12 because $K$ builds $W$ in $\mathscr{D}(P)$; this is where the virtual smallness of $K$ over $P$ comes in. Thus, by restriction, $N$ builds $R \otimes P W$ in $\mathscr{D}(R)$. It remains to note that the complex of $R$-modules $R \otimes_{P} W$ is small, by base change, and that it is non-trivial, for example, by 3.16 (c). This settles our claim.

To complete the proof, it remains to check that the Koszul complex $K$ of $Q$ is virtually small over $P$. However, $Q$ is regular, by construction, so $K \simeq l$ in $\mathscr{D}(Q)$, and hence also in $\mathscr{D}(P)$. The action of $P$ on $l$ factors through $k$, and $k$ is a direct summand of $l$ as a $k$-vector space, and so also as an $P$-module. Thus, $l$ finitely builds $k$ in $\mathscr{D}(P)$; but then, $P$ being regular, $k$ is small.

Here is one corollary. Note that it uses only the special case of the preceding theorem when the residue field extension is finite, so one could also provide a more concise and direct proof.

4.10 Corollary. Let $R$ be a local ring and $M$ a complex of $R$-modules with $\mathrm{H}(M)$ either finite or artinian. When $\operatorname{fd}_{R} M$ is finite, in particular, when $R$ is regular, $M$ is proxy small.

Proof. One may assume that $\mathrm{H}(M) \neq 0$. When $\mathrm{H}(M)$ is finite, the finiteness of $\mathrm{fd}_{R} M$ yields that $M$ is small, by 3.8 , and hence also proxy small. When $\mathrm{H}(M)$ is artinian and $\operatorname{fd}_{R} M$ finite, $M$ is virtually small, by the special case $\varphi=\mathrm{id}^{R}$ of Theorem 4.9, and then its witness is the Koszul complex $K$ of $R$; see Proposition 4.5. To deduce that $M$ is proxy small, it suffices to prove that $M$ is built from $K$. One way to see this is to invoke 3.17 since $\operatorname{supp} K=\{\mathfrak{m}\}=\operatorname{supp} M$, where $\mathfrak{m}$ is the maximal ideal of $R$; the first equality is by 3.16 (a), and the second is obtained by a direct calculation, keeping in mind that $\mathrm{H}(M)$ is artinian. 
Over regular local rings, and for virtual smallness, one has a more drastic result.

4.11 Proposition. Let $(R, \mathfrak{m}, k)$ be a regular local ring. A complex of $R$-modules $M$ is virtually small if and only if $\mathfrak{m} \in \operatorname{supp} M$.

Proof. Indeed, when $M$ is virtually small, Proposition 4.5 yields that $\mathfrak{m}$ is in $\operatorname{supp} M$. The converse is contained in the diagram

$$
M \underset{R}{\Longrightarrow}\left(M \otimes_{R}^{\mathbf{L}} k\right) \underset{R}{\Longrightarrow} k
$$

where the first implication is by 3.12 , since $k$ is finitely built from $R$. As to the second, 2.6 yields that $M \otimes_{R}^{\mathbf{L}} k$ is isomorphic in $\mathscr{D}(R)$ to a graded $k$-vector space, while our hypothesis ensures that this graded $k$-vector space is non-zero, and in particular, that $k$ is a direct summand.

4.12 Remark. Another corollary to Theorem 4.9 is that any small complex of $\widehat{R}$ modules, where $\widehat{R}$ is the $\mathfrak{m}$-adic completion of $R$, is virtually small over $R$. This is subsumed in Corollary 7.5 that asserts: any complex of $\widehat{R}$-modules which is virtually small over $\widehat{R}$ is virtually small over $R$.

Next we focus on differences between proxy smallness and virtual smallness.

\subsection{Proposition. The $R$-module $\widehat{R}$ is proxy small if and only if $R$ is complete.}

Proof. The non-trivial implication is that when $\widehat{R}$ is proxy small, $R$ is complete. Viewed as an $R$-module, the support of $\widehat{R}$ is Spec $R$; this is because it is faithfully flat. Thus, Proposition 4.4 implies that $R$ must be a witness to the proxy smallness of $\widehat{R}$, that is to say, $R$ belongs to Thick ${ }_{R}[\widehat{R}]$. Now consider $\mathbf{L} \Lambda^{\mathfrak{m}}(-)$, the left derived functor of the $\mathfrak{m}$-adic completion functor; for details, see Greenlees-May [16] or Lipman [22], and let $\epsilon:$ id $\rightarrow \mathbf{L} \Lambda^{\mathfrak{m}}(-)$ the corresponding natural transformation. It is not hard to verify that the full subcategory of $\mathscr{D}(R)$ with objects

$$
\mathcal{C}^{\mathfrak{m}}(R)=\left\{M \in \mathscr{D}(R) \mid \epsilon(M): M \rightarrow \mathbf{L} \Lambda^{\mathfrak{m}}(M) \text { is an isomorphism }\right\}
$$

is thick; this is the class of m-adically complete complexes of $R$-modules. Therefore, $\widehat{R}$ being complete, the thick subcategory it generates is contained in $\mathcal{C}^{\mathfrak{m}}(R)$. In particular, $R \in \mathcal{C}^{\mathfrak{m}}(R)$, that is to say, $R$ is complete.

The preceding result, in conjunction with the Remark 4.12, shows that the class of virtually small complexes is considerable larger than the class of proxy small complexes. On the flip side, the latter class is stable, as the former class is not, under important functors on the derived category, as is evidenced by the next result. In it, $\mathbf{R} \Gamma_{I}(M)$ denotes the local cohomology of $M$ supported on $I$; see [22]. 
4.14 Theorem. Let $I$ be an ideal in noetherian ring $R$. When a complex $M$ of $R$-modules is proxy small, so are $\mathbf{R} \Gamma_{I}(M)$ and $\mathbf{L} \Lambda^{I}(M)$.

Proof. We treat the case of local cohomology; completions can be treated along the same lines. Let $W$ be a witness that $M$ is proxy small, and let $K$ be the Koszul complex on a finite set of generators for the ideal $I$. We claim that $K \otimes_{R}^{\mathbf{L}} W$ is a witness that $\mathbf{R} \Gamma_{I}(M)$ is proxy small.

Indeed, $K \otimes_{R}^{\mathbf{L}} W$ is small, for example, by 3.12, because both $K$ and $W$ are small. It is also finitely built from $\mathbf{R} \Gamma_{I}(M)$ as can be seen by following the path from the north-west to the south-east corners of the diagram

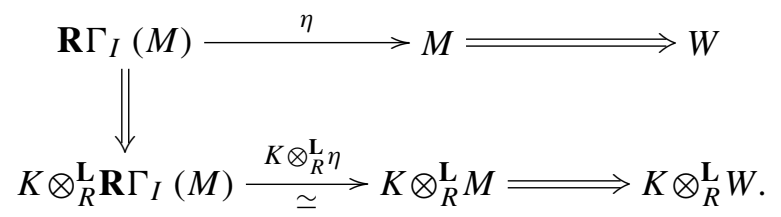

where $\eta$ is the canonical morphism. The implication in the bottom row is induced by the one in the top, while the vertical implication holds because $R \Longrightarrow K$; both these deductions use 3.12. To see that $K \otimes_{R}^{\mathbf{L}} \eta$ is an isomorphism, note the isomorphisms

$$
K \otimes_{R}^{\mathbf{L}} \mathbf{R} \Gamma_{I}(M) \simeq \mathbf{R} \Gamma_{I}(K) \otimes_{R}^{\mathbf{L}} M \quad \text { and } \quad \mathbf{R} \Gamma_{I}(K) \simeq K
$$

where the first can be obtained from [22, (3.3.1)], and the second from [22, (3.2.1)].

It remains to verify that $\mathbf{R} \Gamma_{I}(M)$ is built from $K \otimes_{R}^{\mathbf{L}} W$. Keeping in mind that $M$ and $W$ are built from each other, one has equalities

$$
\operatorname{supp}\left(K \otimes_{R}^{\mathbf{L}} W\right)=\operatorname{supp} K \cap \operatorname{supp} W=\mathrm{V}(I) \cap \operatorname{supp} M=\operatorname{supp} \mathbf{R} \Gamma_{I}(M)
$$

where the first equality is by 3.16 (c), the second combines 3.16 (a) and 3.16 (b), and the last one is by $[22,(3.1 .2)]$ and 3.16 (c). It remains to invoke 3.17 .

It is easy to construct examples that show that the statement on virtual smallness corresponding to the one above does not hold.

4.15 Example. Let $R$ be a noetherian ring and let $I$ and $J$ be proper ideals in $R$ such that $R / I$ is not virtually small, $R / J$ is virtually small, and $\mathrm{V}(I) \cap \mathrm{V}(J)=\emptyset$. The $R$-module $M=R / I \oplus R / J$ is virtually small, since its direct summand $R / J$ has that property, whilst $\mathbf{R} \Gamma_{I}(M) \simeq R / I$ is not.

For instance, let $k$ be a field and $R=k[x, y, z] /\left(x^{2}, x y, y^{2}\right)$. Pick a non-zero element $a \in k$ and consider the ideals $I=(y, z)$ and $J=(x, y, z-a)$ in $R$; evidently, $\mathrm{V}(I) \cap \mathrm{V}(J)=\emptyset$. It follows from Example 4.8 that $R / I$ is not virtually small. On the other hand, $R / J \cong k$, so it is virtually small, with witness the Koszul complex on $\{x, y, z-a\}$. 
The construction above points the way to the analogue of Theorem 4.14 for virtual smallness.

4.16 Theorem. Let $I$ be an ideal in a local ring $R$. If a complex $M$ of $R$-modules is virtually small, then so is $\mathbf{R} \Gamma_{I}(M)$; the converse holds if $\mathbf{R} \Gamma_{I}(M) \nsucceq 0$. The corresponding statement for $\mathbf{L} \Lambda^{I}(M)$ also holds.

Proof. Once again, we only provide details for the statement concerning local cohomology. Let $K$ be the Koszul complex on a finite set of generators for $I$. The crucial point in the proof is that if a complex $W$ is small with $\mathrm{H}(W) \neq 0$, then the complex $K \otimes_{R}^{\mathbf{L}} W$ has the same properties. Indeed, the smallness of $K \otimes_{R}^{\mathbf{L}} W$ follows from 3.12. As to its non-triviality: both $\operatorname{supp} K$ and supp $W$ are non-empty closed subsets of Spec $R$, by 3.16(d), and hence contain the maximal ideal of $R$. Thus, the same is true of $\operatorname{supp}\left(K \otimes_{R}^{\mathbf{L}} W\right)$, by 3.16 (c); this implies $\mathrm{H}\left(K \otimes_{R}^{\mathbf{L}} W\right) \neq 0$.

First we verify that if $M$ is virtually small with witness $W$, then $\mathbf{R} \Gamma_{I}(M)$ is virtually small with witness $K \otimes_{R}^{\mathbf{L}} W$. We may assume that $M \not 0$. Arguing as in the proof of the preceding result, one obtains that $K \otimes_{R}^{\mathbf{L}} W$ is finitely built from $\mathbf{R} \Gamma_{I}(M)$. It remains to note that, by the discussion in the last paragraph, $K \otimes_{R}^{\mathbf{L}} W$ is small and non-zero.

Assume $\mathbf{R} \Gamma_{I}(M)$ is non-zero and virtually small, with witness $W$. Then one has a diagram

$$
M \Longrightarrow\left(K \otimes_{R}^{\mathbf{L}} M\right) \simeq\left(K \otimes_{R}^{\mathbf{L}} \mathbf{R} \Gamma_{I}(M)\right) \Longrightarrow\left(K \otimes_{R}^{\mathbf{L}} W\right)
$$

where the implications are given by 3.12, and the equivalence is verified as in the proof of Theorem 4.14. Since $K \otimes_{R}^{\mathbf{L}} W$ is small and non-trivial, it is a witness that $M$ is virtually small.

To end this section, we present a flat module which is not virtually small.

4.17 Example. Let $R$ be a noetherian ring and $\mathfrak{p}$ a non-maximal prime ideal. We claim that the flat $R$-module $R_{\mathfrak{p}}$ is not virtually small.

Indeed, if $R_{\mathfrak{p}}$ builds a complex $W$, then supp $W \subseteq \operatorname{supp} R_{\mathfrak{p}}$. If $W$ is small, then supp $W$ is a closed subset of Spec $R$, and hence supp $W \nsubseteq \operatorname{supp} R_{\mathfrak{p}}$. Thus, Thick ${ }_{R}\left[R_{\mathfrak{p}}\right]$ contains no small complexes, except those that are homologically zero.

\section{Virtually small complexes as test objects}

In this section $(R, \mathfrak{m}, k)$ is a local ring. Many ring theoretic properties of $R$ can be detected by the finiteness of suitable homological dimensions of some 'test object'. Typically, either $R$ or $k$ plays this role. For instance, the ring $R$ is Gorenstein precisely when it has finite injective dimension when viewed as a module over itself, while $R$ is regular precisely when $k$ has finite flat dimension. 
The main goal of this section, and the next one, is to demonstrate that, often, any virtually small complex can be used as a test object. Specialized to the situation when the complex is small or the residue field, some of these recover well-known results in the literature; however, even then the proofs we present are new. In each case, the crux of the argument is similar, so we describe it in a sufficiently general scenario.

5.1. Let $\lambda$ be a numerical invariant defined on $\mathscr{D}(R)$. The guiding examples are as follows.

(a) Let $X$ be a complex of $R$-modules. For each complex $M$ in $\mathscr{D}(R)$, set

$$
\lambda(M)=\operatorname{amp}\left(X \otimes_{R}^{\mathbf{L}} M\right) .
$$

In the next two examples, $\psi: Q \rightarrow R$ is a local homomorphism; keep in mind the special case where $\psi$ is the identity on $R$.

(b) For each complex $M$ of $R$-modules, set $\lambda(M)=\mathrm{fd}_{Q} M$.

One has also the version involving injective dimensions:

(c) For each complex $M$ of $R$-modules, set $\lambda(X)=\operatorname{id}_{Q} X$.

To a given invariant $\lambda$, we associate the full subcategory of $\mathscr{D}(R)$ defined by

$$
\mathscr{D}^{\lambda}(R)=\{X \in \mathscr{D}(R) \mid \lambda(X)<\infty\} .
$$

Here is a noteworthy feature of examples (a)-(c) above: $\mathscr{D}^{\lambda}(R)$ is a thick subcategory of $\mathscr{D}(R)$. The required arguments are a simple extension of those required for verifying the assertions in 3.2 , which correspond to the special case $\psi=\mathrm{id}^{R}$.

We record the following principle that is used repeatedly in this section. It is evident that there is a more general version, dealing with properties of complexes other than the finiteness of some numerical invariant. However, we have opted to state it in its simplest form for it is adequate for our present purposes.

5.2. Principle. Let $K$ be the Koszul complex on a finite set of generators of $\mathfrak{m}$. Suppose $\lambda$ has the following properties:

(a) $\mathscr{D}^{\lambda}(R)$ is a thick subcategory of $\mathscr{D}(R)$;

(b) if $\lambda(K)$ is finite, then so is $\lambda(R)$.

If $M$ is virtually small, with $\mathrm{H}(M) \neq 0$, and $\lambda(M)$ is finite, then $\lambda(R)$ is finite.

Indeed, when $\lambda(M)$ is finite, $\mathscr{D}^{\lambda}(R)$ contains $M$, and hence also $\operatorname{Thick}_{R}[M]$, by hypothesis (a). If $M$ is virtually small, then $K$ lies in $\operatorname{Thick}_{R}[M]$, by 4.5 , so we conclude that $K$ lies in $\mathscr{D}^{\lambda}(R)$, that is to say, $\lambda(K)$ is finite. Now hypothesis (b) yields the desired conclusion.

The rest of this section provides diverse applications of this proof-principle. 
Homological dimensions. The theorem below unifies and extends numerous characterizations of Gorenstein rings. The equivalence of (a) and (b) is [7, (13.2)], while (c) $\Longrightarrow$ (a), applied to $\psi=\mathrm{id}^{R}$, contains the well-known result that when $R$ has a homologically finite complex of finite injective dimension and finite flat dimension, then it is Gorenstein; see also Corollary 5.4 below.

5.3 Theorem. Let $\psi: Q \rightarrow R$ be a local homomorphism. The following conditions are equivalent.

(a) $Q$ is Gorenstein and $\mathrm{fd}_{Q} R$ is finite;

(b) $\operatorname{id}_{Q} R$ is finite;

(c) $\operatorname{id}_{Q} M$ is finite for a virtually small complex $M$ over $R$, with $\mathrm{H}(M) \neq 0$.

Proof. As noted before, (a) $\Longleftrightarrow$ (b) is [7, (13.2)]. Taking $M=R$ settles (b) $\Longrightarrow$ (c), so it remains to verify that (c) implies (b).

For each complex $X$ of $R$-modules, set $\lambda(X)=\operatorname{id}_{Q} X$. As noted in 5.1, the subcategory $\mathscr{D}^{\lambda}(R)$ of $\mathscr{D}(R)$ is thick. In order to apply Principle 5.2 to the desired end, it remains to verify that when the Koszul complex $K$ of $R$ has finite injective dimension over $Q$, so does $R$. With $h$ the residue field of $Q$, in $\mathscr{D}(R)$ one has the associativity isomorphism

$$
\mathbf{R H o m}_{Q}(h, K) \simeq \mathbf{R H o m}_{Q}(h, R) \otimes_{R}^{\mathbf{L}} K .
$$

The complex $\mathbf{R H o m}_{Q}(h, R)$ of $R$-modules is homologically degreewise finite, so the isomorphism above and 2.9(d) imply: $\sup \mathbf{R} \operatorname{Hom}_{Q}(h, K)$ and $\sup \mathbf{R H o m}_{Q}(h, R)$ are simultaneously finite. It remains to note that for any homologically finite complex $X$ of $R$-modules, one has $\operatorname{id}_{Q} X=\sup \mathbf{R H o m}_{Q}(h, X)$, by [3, (5.5.I)].

One corollary is the following characterization Gorenstein rings. We do not claim it is new; only that it illustrates the scope of the preceding result.

5.4 Corollary. A local ring $(R, \mathfrak{m}, k)$ is Gorenstein if and only if the flat dimension of the injective hull of $k$ is finite.

Proof. Let $E$ be the injective hull of $k$. When $R$ is Gorenstein, $\operatorname{fd}_{R} E$ is finite, since $\operatorname{id}_{R} E$ is finite; see [11, (3.3.4)]. Conversely, since $E$ is artinian, if $\mathrm{fd}_{R} E$ is finite, then it is virtually small, by Corollary 4.10 , so it remains to invoke (c) $\Longrightarrow$ (a) of Theorem 5.3.

The next result is an analogue for flat dimensions of (c) $\Longrightarrow$ (b) in 5.3.

5.5 Theorem. Let $\psi: Q \rightarrow R$ be a local homomorphism and $M$ a virtually small complex of $R$-modules with $\mathrm{H}(M) \neq 0$. If $\mathrm{fd}_{Q} M$ is finite, then $\mathrm{fd}_{Q} R$ is finite. 
5.6 Remark. Let $R \rightarrow S$ be another local homomorphism and $N$ a homologically finite complex of $S$-modules with $\operatorname{fd}_{R} N$ finite. Then $N$ is virtually small over $R$, by Theorem 4.9, so the preceding result yields: when $\operatorname{fd}_{Q} N$ is finite, so is $\operatorname{fd}_{Q} R$. In this way we recover $[15,(3.2)]$.

Proof. The proof is similar to that of (c) $\Longrightarrow$ (b) in Theorem 5.3; the details are given for the sake of completeness. For each complex $X$ of $R$-modules, set $\lambda(X)=\mathrm{fd}_{Q} X$; note that the subcategory $\mathscr{D}^{\lambda}(R)$ of $\mathscr{D}(R)$, introduced in 5.1 , is thick. Let $K$ be the Koszul complex of $R$, and $h$ the residue field of $Q$. One has the associativity isomorphism

$$
h \otimes_{Q}^{\mathbf{L}} K \simeq\left(h \otimes_{Q}^{\mathbf{L}} R\right) \otimes_{R}^{\mathbf{L}} K .
$$

The $R$-module $\mathrm{H}\left(h \otimes_{Q}^{\mathbf{L}} R\right)$ is degreewise finite, so it follows from the isomorphism above and $2.9(\mathrm{~d})$ that $\sup \left(h \otimes_{Q}^{\mathbf{L}} K\right)$ and $\sup \left(h \otimes_{Q}^{\mathbf{L}} R\right)$ are simultaneously finite. Thus, [3, (5.5)] implies that $\operatorname{fd}_{Q} K$ and $\mathrm{fd}_{Q} R$ are simultaneously finite. Principle 5.2 now yields the desired conclusion.

We record a direct corollary.

5.7 Corollary. Let $Q \stackrel{\psi}{\longrightarrow} R \stackrel{\pi}{\longrightarrow} Q$ be local homomorphisms with $\pi \circ \psi=\mathrm{id}^{Q}$. If $Q$ viewed as an $R$-module via $\pi$ is virtually small, then $\operatorname{fd}_{Q} R$ is finite.

This result is useful for detecting some complexes that are not virtually small.

5.8 Example. Let $k$ be a field, $R=k[x, y] /\left(x^{2}, x y, y^{2}\right)$. As claimed in Example 4.8, the $R$-module $R /(y)=k[x] /\left(x^{2}\right)$ is not virtually small.

Here is a justification: set $Q=k[x] /\left(x^{2}\right)$, let $\psi: Q \rightarrow R$ be the natural inclusion, and $\pi: R \rightarrow Q$ the canonical surjection; evidently, $\pi \circ \psi=\mathrm{id}^{Q}$. It is easy to see that $\mathrm{fd}_{Q} R=\infty$; for example, since $Q$ is zero dimensional, $\mathrm{fd}_{Q} R$ finite implies, due to the Auslander-Buchsbaum Equality, that $R$ is a free $Q$-module, which it is not. Thus, Corollary 5.7 yields the desired conclusion.

Next we turn to asymptotic invariants over local homomorphisms.

Complexity and curvature. Let $\psi:(Q, \mathfrak{q}, h) \rightarrow(R, \mathfrak{m}, k)$ be a local homomorphism and $M$ a homologically finite complex of $R$-modules. Let $x \subset R$ be a minimal set generating the maximal ideal of $R / \mathfrak{q} R$.

Following Avramov, Iyengar, and Miller [7] we introduce, for each integer $n$, the $n$th Betti number, respectively, the $n$th Bass number, of $M$ over $\psi$ to be the number

respectively

$$
\beta_{n}^{\psi}(M)=\operatorname{rank}_{k} \operatorname{Tor}_{n}^{Q}(h, \mathrm{~K}[\boldsymbol{x} ; M]),
$$

$$
\mu_{\psi}^{n}(M)=\operatorname{rank}_{k} \operatorname{Ext}_{Q}^{n}(h, \mathrm{~K}[\boldsymbol{x} ; M]) .
$$


Note that when $\psi=\mathrm{id}^{R}$, these coincide with the classical Betti numbers and Bass numbers of $M$ over $R$. In [7], the reader will find an in depth study of invariants over $\psi$, as well as of the ones below which reflect their asymptotic behaviour.

The complexity of $M$ over $\psi$ is the number

$$
\operatorname{cx}_{\psi} M=\inf \left\{\begin{array}{l|l}
d \in \mathbb{N} & \begin{array}{l}
\text { there exists a number } c \in \mathbb{R} \text { such } \\
\text { that } \beta_{n}^{\psi}(M) \leq c n^{d-1} \text { for all } n \gg 0
\end{array}
\end{array}\right\} .
$$

The curvature of $M$ over $\psi$ is the number

$$
\operatorname{curv}_{\psi} M=\limsup _{n} \sqrt[n]{\beta_{n}^{\psi}(M)} .
$$

Replacing Betti numbers by Bass numbers one obtains the injective complexity $\operatorname{injcx}_{\psi} M$, and the injective curvature injcurv ${ }_{\psi} M$, of $M$ over $\varphi$.

5.9 Lemma. Let $M$ be a homologically finite complex of $R$-modules. For each complex $X$ in Thick $_{R}[M]$, the following inequalities hold:

$$
\operatorname{cx}_{\psi} X \leq \operatorname{cx}_{\psi} M \text { and } \operatorname{curv}_{\psi} X \leq \operatorname{curv}_{\psi} M .
$$

Proof. We give the argument for the claim about complexity; the one for curvature is similar. The key point is to verify that

$$
\mathcal{T}=\left\{X \in \mathscr{D}(R) \mid X \text { is homologically finite and } \mathrm{cx}_{\psi} X \leq \mathrm{cx}_{\psi} M\right\}
$$

is a thick subcategory of $\mathscr{D}(R)$. For then, since $M$ is in $\mathcal{T}$, so would anything in $\operatorname{Thick}_{R}[M]$, which is the desired result.

Let $\boldsymbol{x} \subset R$ be a minimal set generating the maximal ideal of $R / \mathfrak{q} R$.

When $X \simeq Y$ it is immediate that $X$ and $Y$ are in $\mathcal{T}$ simultaneously. In $\mathscr{D}(R)$, if $X$ is a direct summand of $Y$, then $h \otimes_{Q}^{\mathbf{L}} \mathrm{K}[\boldsymbol{x} ; X]$ is a direct summand of $h \otimes_{Q}^{\mathbf{L}} \mathrm{K}[\boldsymbol{x} ; Y]$. Thus, passing to homology, for each integer $n$ one obtains

$$
\operatorname{rank}_{k} \operatorname{Tor}_{n}^{Q}(h, \mathrm{~K}[\boldsymbol{x} ; X]) \leq \operatorname{rank}_{k} \operatorname{Tor}_{n}^{Q}(h, \mathrm{~K}[\boldsymbol{x} ; Y])
$$

that is to say, $\beta_{n}^{\psi}(X) \leq \beta_{n}^{\psi}(Y)$. This yields $\operatorname{cx}_{\psi} X \leq \operatorname{cx}_{\psi} Y$, hence when $Y$ is in $\mathcal{T}$, so is $X$. Thus, it remains to establish that in $\mathscr{D}(R)$, given an exact triangle

$$
X \longrightarrow Y \longrightarrow Z \longrightarrow \Sigma X
$$

when any two of $X, Y$, or $Z$ are in $\mathcal{T}$, so is the third. To see this, note that applying $\mathrm{K}[\boldsymbol{x} ; R] \otimes_{R}-$ and then $h \otimes_{Q}^{\mathbf{L}}-$ to the triangle above, and passing to homology leads to a long exact sequence

$$
\begin{gathered}
\cdots \rightarrow \operatorname{Tor}_{n}^{Q}(h, \mathrm{~K}[\boldsymbol{x} ; X]) \rightarrow \operatorname{Tor}_{n}^{Q}(h, \mathrm{~K}[\boldsymbol{x} ; Y]) \rightarrow \operatorname{Tor}_{n}^{Q}(h, \mathrm{~K}[\boldsymbol{x} ; Z]) \rightarrow \\
\rightarrow \operatorname{Tor}_{n-1}^{Q}(h, \mathrm{~K}[\boldsymbol{x} ; X]) \rightarrow \cdots
\end{gathered}
$$


of $k$-vector spaces. Thus, computing ranks yields inequalities

$$
\begin{aligned}
& \beta_{n}^{\psi}(X) \leq \beta_{n}^{\psi}(Y)+\beta_{n+1}^{\psi}(Z), \\
& \beta_{n}^{\psi}(Y) \leq \beta_{n}^{\psi}(X)+\beta_{n}^{\psi}(Z), \\
& \beta_{n}^{\psi}(Z) \leq \beta_{n-1}^{\psi}(X)+\beta_{n}^{\psi}(Y) .
\end{aligned}
$$

From these it is clear that if any two of $\mathrm{cx}_{\psi} X, \mathrm{cx}_{\psi} Y$, or $\mathrm{cx}_{\psi} Z$ are less than $\mathrm{cx}_{\psi} M$, then so is the third. Thus, the subcategory $\mathcal{T}$ is thick, as desired.

5.10 Theorem. Let $\psi: Q \rightarrow R$ be a local homomorphism and $M$ a virtually small complex of $R$-modules, with $\mathrm{H}(M)$ finite. Each homologically finite complex of $R$ modules $Y$ satisfies inequalities:

$$
\operatorname{cx}_{\psi} Y \leq \operatorname{cx}_{R} Y+\operatorname{cx}_{\psi} M \text { and } \operatorname{curv}_{\psi} Y \leq \max \left\{\operatorname{curv}_{R} Y, \operatorname{curv}_{\psi} M\right\} .
$$

In particular, $\mathrm{cx}_{\psi} R \leq \mathrm{cx}_{\psi} M$ and $\operatorname{curv}_{\psi} R \leq \operatorname{curv}_{\psi} M$.

5.11 Remarks. One has also the following injective analogues of the theorem above:

$$
\operatorname{injcx}_{\psi} Y \leq \operatorname{cx}_{R} Y+\operatorname{injcx}_{\psi} M \text { and } \operatorname{injcurv}_{\psi} Y \leq \max \left\{\operatorname{curv}_{R} Y, \operatorname{injcurv}_{\psi} M\right\} \text {. }
$$

The argument is similar to the one for complexity and curvature.

Another remark is that a homologically finite complex of $R$-modules has finite flat dimension over $Q$ if and only if its complexity over $\psi$ is zero; see [7, (7.1.3.1)]. Thus, the theorem above is a quantitative extension of Theorem 5.5, restricted however to homologically finite complexes.

Proof of Theorem 5.10. Applying [7, (9.1.1.1)], with $\varphi=\mathrm{id}^{R}$, yields

$$
\mathrm{cx}_{\psi} Y \leq \operatorname{cx}_{R} Y+\mathrm{cx}_{\psi} R \text { and } \operatorname{curv}_{\psi} Y \leq \max \left\{\operatorname{curv}_{R} Y, \operatorname{curv}_{\psi} R\right\} .
$$

Thus, it suffices to verify the desired inequalities in the special case $Y=R$. Since $M$ is virtually small, $\operatorname{Thick}_{R}[M]$ contains $K$, the Koszul complex of $R$; this is by Proposition 4.5. Therefore, Lemma 5.9 yields inequalities

$$
\operatorname{cx}_{\psi} K \leq \operatorname{cx}_{\psi} M \quad \text { and } \quad \operatorname{curv}_{\psi} K \leq \operatorname{curv}_{\psi} M .
$$

It remains to note that $\mathrm{cx}_{\psi} K=\mathrm{cx}_{\psi} R$ and $\operatorname{curv}_{\psi} K=\operatorname{curv}_{\psi} R$, by [7, (7.2.1)].

The preceding theorem has implications for the descent of the complete intersection property along local homomorphisms; see Theorem 9.7. 
Finiteness of amplitudes. Let $R$ be a local ring and $M$ a small complex of $R$ modules. Iversen [19] has proved that for each homologically finite complex $Y$ of $R$-modules, one has the Amplitude Inequality

$$
\operatorname{amp}(Y) \leq \operatorname{amp}\left(M \otimes_{R}^{\mathbf{L}} Y\right) .
$$

In fact, Iversen established that it is equivalent to the New Intersection Theorem, which had then been verified only for rings containing fields. Since then, Paul Roberts [25], has proved that the latter result, and hence also the Amplitude Inequality, is valid for all local rings.

It was proved in [15] that the Amplitude Inequality is valid even when $\mathrm{H}(Y)$ is only degreewise finite; no boundedness assumptions are required. In the light of Iversen's result, this amounted to proving that when $\operatorname{amp}\left(M \otimes_{R}^{\mathbf{L}} Y\right)$ is finite, so is $\operatorname{amp}(Y)$. Numerous examples were given in [15], see also [20], to illustrate the utility of this unbounded version of the Amplitude Inequality.

The theorem below, in conjunction with Theorem 4.9, subsumes [15, Theorem III]. Our approach differs from the one in [15], and yields a concise, more transparent, proof even in the special case where $M$ is a small complex.

5.12 Theorem. Let $R$ be a local ring and let $M$ be a virtually small complex of $R$ modules with $\mathrm{H}(M) \neq 0$. Let $Y$ be a complex of $R$-modules with $\mathrm{H}(Y)$ degreewise finite.

If $\operatorname{amp}\left(M \otimes_{R}^{\mathbf{L}} Y\right)$ is finite, then $\operatorname{amp}(Y)$ is finite as well.

Proof. For each complex $X$ of $R$-modules, set $\lambda(X)=\operatorname{amp}\left(X \otimes_{R}^{\mathbf{L}} Y\right)$; thus, the desired result is that $\lambda(R)$ is finite. To obtain this, we check that the conditions in 5.2 are satisfied: (a) is a routine verification, while (b) is contained in 2.9 (d).

5.13 Remark. The conclusion of the theorem above cannot be strengthened to an inequality: $\operatorname{amp}(Y) \leq \operatorname{amp}\left(M \otimes_{R}^{\mathbf{L}} Y\right)$. Indeed, set $R=k[x, y] /(x y)$, let $M=R /(y)$ and $Y$ the complex

$$
0 \rightarrow R \stackrel{x}{\longrightarrow} R \rightarrow 0 .
$$

The ring $R$ is a complete intersection and $\mathrm{H}(M)$ is finite, so Theorem 9.4 yields $M$ is virtually small. However, $\operatorname{amp}(Y)=1$ whilst $\operatorname{amp}\left(M \otimes_{R}^{\mathbf{L}} Y\right)=0$.

\section{Gorenstein dimensions}

Let $(R, \mathfrak{m}, k)$ be a local ring and $M$ a complex of $R$-modules with $\mathrm{H}(M)$ degreewise finite and bounded below. We denote G- $\operatorname{dim}_{R} M$ the Gorenstein dimension, often abbreviated to $G$-dimension, of $M$ over $R$. The reader may consult Christensen's 
monograph [11] for details; there, to be precise, in [11, (2.3.8)], one finds also the following characterization of complexes of finite G-dimension that is most apposite for us. It makes it clear that every small complex has finite $G$-dimension.

6.1. A homologically finite complex $M$ has finite $G$-dimension if and only if

(a) $\operatorname{RHom}_{R}(M, R)$ is homologically bounded, and

(b) the canonical biduality morphism is an isomorphism:

$$
R \stackrel{\simeq}{\longrightarrow} \operatorname{RHom}_{R}\left(\operatorname{RHom}_{R}(M, R), R\right) .
$$

When this is the case, $\mathrm{G}-\operatorname{dim}_{R} M=-\inf \operatorname{RHom}_{R}(M, R)$.

First, we present a new characterization of small complexes; this is in preparation for Theorem 6.7, and the hypotheses are dictated by it. Note that condition (ii) in the result below is satisfied when $M$ is virtually small, since a small complex has finite G-dimension. One may thus view it as a test for detecting small complexes from among virtually small ones.

6.2 Theorem. Let $R$ be a local ring. Each homologically degreewise finite and bounded below complex $M$ of R-modules satisfying the following conditions is small:

(i) $\operatorname{RHom}_{R}(M, M)$ is small;

(ii) $\operatorname{Thick}_{R}[M]$ has a homologically non-zero complex of finite $G$-dimension.

If, in addition, $\mathbf{R H o m}_{R}(M, M) \simeq R$, then $M \simeq \Sigma^{d} R$ for some integer $d$.

6.3 Remarks. A homologically finite complex $M$ with $\mathbf{R H o m}_{R}(M, M) \simeq R$ is said to be semi-dualizing. For example, $R$ is semi-dualizing, as is a dualizing complex for $R$ : a semi-dualizing complex of finite injective dimension. The result above implies that a semi-dualizing complex of finite $G$-dimension is isomorphic to $\Sigma^{d} R$, for some $d \in \mathbb{Z}$; this is contained in [10, (8.3)].

Theorem 6.2 suggests that it would be fruitful to study those complexes $M$ for which Thick ${ }_{R}[M]$ contains a complex of finite $G$-dimension.

The proof is given in 6.5 below. It uses an elementary remark concerning minimal resolutions.

6.4. The Poincaré series and the Bass series of $M$ are the formal Laurent series

$$
\begin{aligned}
\mathrm{P}_{M}^{R}(t) & =\sum_{n \in \mathrm{Z}} \operatorname{rank}_{k} \operatorname{Tor}_{n}^{R}(k, M) \cdot t^{n}, \\
\mathrm{I}_{R}^{M}(t) & =\sum_{n \in \mathrm{Z}} \operatorname{rank}_{k} \operatorname{Ext}_{R}^{n}(k, M) \cdot t^{n} .
\end{aligned}
$$


When there is no confusion about the ambient ring, we drop it from the subscript and the superscript. As noted in 2.3, the complex $M$ is small if and only if its minimal resolution is finite, which translates to the condition that $\mathrm{P}_{M}(t)$ is a Laurent polynomial. In the sequel, it is useful to keep in mind the following stronger, but no more difficult to establish, version of this remark: $\mathrm{P}_{M}(t)=\sum_{n \in \mathrm{Z}} b_{n} t^{n}$ if and only if $M$ is isomorphic to a complex of the form

$$
F:=\cdots \rightarrow R^{b_{n+1}} \rightarrow R^{b_{n}} \rightarrow R^{b_{n-1}} \rightarrow \cdots .
$$

where $R^{b_{n}}$ sits in homological degree $n$, and $\partial(F) \subseteq \mathfrak{m} F$.

6.5. Proof of Theorem 6.2. By (ii), in $\operatorname{Thick}_{R}[M]$ there is a complex $G$ of $R$-modules with $\mathrm{H}(G) \neq 0$ of finite $\mathrm{G}$-dimension.

First we prove that $G$ is small. To this end, note that 3.12 yields

$$
\operatorname{RHom}_{R}(M, M) \underset{R}{\Longrightarrow} \operatorname{RHom}_{R}(M, G) \underset{R}{\Longrightarrow} \operatorname{RHom}_{R}(G, G) \text {. }
$$

Hence $\mathbf{R H o m}_{R}(G, G)$ is finitely built from $\mathbf{R H o m}_{R}(M, M)$, so $\mathbf{R H o m}_{R}(G, G)$ is also small; here one is using 3.9. By definition 6.1, the biduality morphism associated to $G$ is an isomorphism, and this leads to the first of the isomorphisms:

$$
\begin{aligned}
\mathbf{R H o m}_{R}(k, G) & \simeq \mathbf{R H o m}_{R}\left(k, \mathbf{R H o m}_{R}\left(\mathbf{R H o m}_{R}(G, R), R\right)\right) \\
& \simeq \mathbf{R H o m}_{R}\left(\mathbf{R H o m}_{R}(G, R) \otimes \mathbf{L}_{R}^{\mathbf{L}} k, R\right) \\
& \simeq \mathbf{R H o m}_{k}\left(\mathbf{R H o m}_{R}(G, R) \otimes_{R}^{\mathbf{L}} k, \mathbf{R H o m}_{R}(k, R)\right) .
\end{aligned}
$$

The second and the third isomorphisms are by adjunction; the last one uses also 2.6. Set $G^{*}=\mathbf{R H o m}_{R}(G, R)$; this complex is homologically finite since the G-dimension of $G$ is finite, so the isomorphisms above yield an equality of formal Laurent series

$$
\mathrm{I}^{G}(t)=\mathrm{P}_{G^{*}}(t) \mathrm{I}^{R}(t) .
$$

Set $\mathscr{E}=\mathbf{R H o m}_{R}(G, G)$, and consider now the following isomorphisms

$$
\begin{aligned}
\mathbf{R H o m}_{R}(k, \mathcal{E}) & \simeq \mathbf{R H o m}_{R}(k, R) \otimes_{R}^{\mathbf{L}} \mathcal{E} \\
& \simeq \mathbf{R H o m}_{R}(k, R) \otimes_{k}^{\mathbf{L}}\left(k \otimes_{R}^{\mathbf{L}} \mathcal{E}\right)
\end{aligned}
$$

where the first one holds because $\mathcal{E}$ is small and the second follows from 2.6 and the associativity of tensor products. On the other hand, adjunction implies that

$$
\begin{aligned}
\operatorname{RHom}_{R}(k, \mathcal{E}) & \simeq \mathbf{R H o m}_{R}\left(G \otimes{ }_{R}^{\mathbf{L}} k, G\right) \\
& \simeq \mathbf{R H o m}_{k}\left(G \otimes_{R}^{\mathbf{L}} k, \mathbf{R H o m}_{R}(k, G)\right) .
\end{aligned}
$$


The two sets of isomorphisms above imply the equality of formal Laurent series:

$$
\mathrm{I}^{R}(t) \mathrm{P}_{\mathcal{E}}(t)=\mathrm{P}_{G}(t) \mathrm{I}^{G}(t) .
$$

Combining the displayed equalities above of formal Laurent series yields

$$
\mathrm{I}^{R}(t) \mathrm{P}_{\mathcal{E}}(t)=\mathrm{P}_{G}(t) \mathrm{P}_{G^{*}}(t) \mathrm{I}^{R}(t) .
$$

Since the ring of Laurent series is a domain, this equality is equivalent to

$$
\mathrm{P}_{\mathcal{E}}(t)=\mathrm{P}_{G}(t) \mathrm{P}_{G^{*}}(t) .
$$

The coefficients of all the Laurent series that appear in the equality are non-negative integers and $\mathrm{P}_{\mathcal{E}}(t)$ is a Laurent polynomial, because $\mathcal{E}$ is small. Thus, $\mathrm{P}_{G}(t)$ must also be a Laurent polynomial, that is to say, $G$ must be small, as desired.

Next we verify that $M$ is small. We know now that the complex $G$ is small, so

$$
\mathbf{R H o m}_{R}(G, M) \simeq \mathbf{R H o m}_{R}(G, R) \otimes_{R}^{\mathbf{L}} M .
$$

On the other hand, $\mathbf{R H o m}_{R}(G, M)$ is finitely built from $\mathbf{R} \operatorname{Hom}_{R}(M, M)$, since $G$ is finitely built from $M$, by hypothesis (ii). Thus, hypothesis (i) and the isomorphism above imply that $\mathbf{R H o m}_{R}(G, R) \otimes \otimes_{R}^{\mathbf{L}} M$ is small. Since the ring $R$ is local and both $\mathbf{R H o m}_{R}(G, R)$ and $M$ are homologically degreewise finite and bounded below - the first because it is small and the second by hypothesis - one deduces that $M$ is small; see 2.5.

Finally, when $\operatorname{RHom}_{R}(M, M) \simeq R$ as well, in the analogue for $M$ of the equality (*) above, one has $\mathrm{P}_{\mathcal{E}}(t)=1$, and that implies $\mathrm{P}_{M}(t)=t^{d}$ for some integer $d$. Therefore, $M \simeq \Sigma^{d} R$, by 6.4 .

In the remainder of this section, $\psi: Q \rightarrow R$ is a local homomorphism.

G-dimension over $\psi$. In [20], Iyengar and Sather-Wagstaff introduce an invariant G- $\operatorname{dim}_{\psi} M$, called the $G$-dimension of $M$ over $\psi$. It equals G-dim ${ }_{R} M$ in the special case when $\psi$ is the identity homomorphism of $R$. In [20], many classical results on G-dimension over local rings are extended to this setting. The result below, contained in $[20,(5.1)]$, is one such.

Theorem. If the complex $M$ of $R$-modules is small, with $\mathrm{H}(M) \neq 0$, and $\mathrm{G}-\operatorname{dim}_{\psi} M$ is finite, then $\mathrm{G}-\operatorname{dim}_{\psi} R$ is finite as well.

This theorem has been an important technical tool in the development in [20]; see, for example, $[20, \S 6]$. This may explain the relevance of the next result, which is analogous to Theorem 5.5. 
6.6 Theorem. Let $\psi: Q \rightarrow R$ be a local homomorphism and $M$ a homologically finite complex of $R$-modules with $\mathrm{H}(M) \neq 0$. If $M$ is virtually small and $\mathrm{G}-\operatorname{dim}_{\psi} M$ is finite, then $\mathrm{G}-\operatorname{dim}_{\psi} R$ is finite.

Sketch of proof. One way to prove this result is to mimic 5.2: first, one observes that the class of homologically finite complexes of $R$-modules with finite G-dimension over $\psi$ is a thick subcategory of $\mathscr{D}(R)$. This can be deduced from the corresponding statement for the classical G-dimension, and it brings us to the case where $M$ equals $K$, the Koszul complex of $R$. Then one can either appeal to [20, (5.1)], or resort to a direct argument along the lines of the one used to prove that (c) $\Longrightarrow$ (a) in Theorem 5.3.

Quasi-Gorenstein homomorphisms. Assume G-dim$\psi(R)$ is finite, and let $\mathrm{I}^{\psi}(t)$ denote the Bass series of $\psi$. This is a formal Laurent series with non-negative integer coefficients that was introduced in [4], as was the notion of a quasi-Gorenstein homomorphism: one for which $\mathrm{I}^{\psi}(t)=t^{d}$ for some $d \in \mathbb{Z}$; see [4, (7.4)].

Suppose now that $\psi$ has a dualizing complex $D$, in the sense of [4]. It follows from $[4,(5.4),(7.8)]$ that $\psi$ is quasi-Gorenstein precisely when $D \simeq \Sigma^{d} R$ for some integer $d$. This characterization of quasi-Gorenstein homomorphisms is significantly generalized in the result below. Besides being of intrinsic interest, it is plays an important role in the development in Section 9; its proof is short only because most of the argument has been absorbed into Theorem 6.2.

6.7 Theorem. Let $\psi: Q \rightarrow R$ be a local homomorphism with $\mathrm{G}-\operatorname{dim}_{\psi} R$ finite, and let $D$ be a dualizing complex for $\psi$. If $\operatorname{Thick}_{R}[D]$ contains a homologically non-zero complex of finite G-dimension, then $\psi$ is quasi-Gorenstein.

Proof. The homothety $R \rightarrow \mathbf{R H o m}_{R}(D, D)$ is an isomorphism; this is one of the defining properties of a dualizing complex for $\psi ;$ cf. $[4, \S 5]$. Thus, when $\operatorname{Thick}_{R}[D]$ has a homologically non-zero complex of finite G-dimension, Theorem 6.2 yields $D \simeq \Sigma^{d} R$ for some $d \in \mathbb{Z}$. As noted above, this entails $\psi$ is quasi-Gorenstein.

6.8 Remark. The case $\psi=\mathrm{id}^{R}$ of the theorem contains a well-known characterization of Gorenstein rings: if $R$ has a dualizing complex of finite G-dimension, then $R$ is Gorenstein; see [11, (3.4.12)].

\section{Descent}

This section returns to the general study of virtually small complexes. We introduce some terminology to facilitate further discussion. Given a homomorphism of rings $\varphi: R \rightarrow S$, we say virtual smallness descends along $\varphi$ if each complex of $S$-modules 
that is virtually small over $S$ is also virtually small over $R$. In the same vein, one speaks of the descent of proxy smallness along $\varphi$. The use of the word 'descent' is keeping in line with the literature in commutative algebra, and may be less perplexing when one views $\varphi$ as an algebraic geometer does: in terms of the induced map

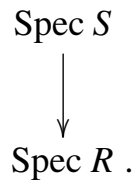

Most of the results in this section are motivated by the following:

7.1 Problem. Identify conditions on $\varphi$ which ensure that virtual smallness descends along $\varphi$.

We also consider the analogous problem for proxy smallness. Our first result is:

7.2 Proposition. Let $\varphi: R \rightarrow S$ be a homomorphism of rings. If $S$ is small as an $R$-module, then both proxy smallness and virtual smallness descend along $\varphi$.

Proof. Let $N$ be proxy small complex of $S$-modules, with witness $W$. Thus, from the properties (i)-(iii) of $W$ in 4.1 one obtains, by restriction 3.13, the corresponding properties of $W$ in $\mathscr{D}(R)$ :

(i) $W$ is finitely built from $S$,

(ii) $W$ is finitely built from $N$, and

(iii) $N$ is built from $W$.

By hypothesis, the $R$-module $S$ is finitely built from $R$, so that (i) and 3.9 imply: $W$ is finitely built from $R$. This, in conjunction with (ii) and (iii) above, yields that $W$ is a witness that $N$ is proxy small over $R$.

A similar argument settles the claim about virtual smallness.

It may be worth drawing attention to the point that the preceding result does not require the rings to be noetherian. With that hypothesis, and the surjectivity of $\varphi$, one can do significantly better, and obtain a perfect 'transitivity' statement. Note that the converses of the statements below also hold; this is a virtually trivial remark.

7.3 Theorem. Let $\varphi: R \rightarrow S$ be a surjective homomorphism of noetherian rings.

If $S$ is proxy small over $R$, then proxy smallness descends along $\varphi$.

If $S$ is virtually small over $R$, then virtual smallness descends along $\varphi$. 
Proof. We verify the claim on proxy smallness; the argument for virtual smallness is similar, and even a tad easier. Let $N$ be a proxy small complex of $S$-modules. By Proposition 4.4, the subset $\operatorname{supp}_{S} N \subseteq \operatorname{Spec} S$ is closed, that it to say, of the form $\mathrm{V}(I)$ for some ideal $I$ of $S$. Pick a finite set $\boldsymbol{x}$ of elements in $R$ that map to a generating set for $I$, and let $K$ be the Koszul complex on $\boldsymbol{x}$. The complex $S \otimes_{R} K$ is the Koszul complex over $S$ on the set $\boldsymbol{x} S$, and hence it is small and its support is $\mathrm{V}(I)$; see 3.16 (a). Thus, Proposition 4.4 implies that it is a witness that $N$ is proxy small in $\mathscr{D}(S)$. With $W$ a witness that $S$ is proxy small over $R$, we claim that the complex of $R$-modules $W \otimes{ }_{R}^{\mathbf{L}} K$ is a witness that $N$ is proxy small in $\mathscr{D}(R)$.

Indeed, $W \otimes_{R}^{\mathbf{L}} K$ is finitely built from $R$, since both $W$ and $K$ are finitely built from $R$; for example, by 3.12. It is also finitely built from $N$ because

$$
N \underset{S}{\Longrightarrow}\left(S \otimes_{R}^{\mathbf{L}} K\right) \quad \text { and } \quad\left(S \otimes_{R}^{\mathbf{L}} K\right) \underset{R}{\Longrightarrow}\left(W \otimes_{R}^{\mathbf{L}} K\right) .
$$

Here the first implication is valid because $S \otimes_{R} K$ is a witness for $N$ in $\mathscr{D}(S)$, and the second follows from 3.12, because $W$ is a witness for $S$ in $\mathscr{D}(R)$. Finally, the tensor product formula for supports 3.16 yields that in $\operatorname{Spec} R$ one has equalities

$$
\operatorname{supp}_{R}\left(W \otimes{ }_{R}^{\mathbf{L}} K\right)=\operatorname{supp}_{R} W \cap \operatorname{supp}_{R} K=\operatorname{supp}_{R} S \cap \operatorname{supp}_{R} N=\operatorname{supp}_{R} N .
$$

Thus, 3.17 implies that $N$ is built from $W \otimes_{R}^{\mathbf{L}} K$, as desired.

Over local homomorphisms, and for virtual smallness, one has the stronger result below; it complements, and its proof is based on, Theorem 4.9.

7.4 Theorem. If $\varphi: R \rightarrow S$ is a local homomorphism with $\operatorname{~fd}_{R} S$ is finite, then virtual smallness descends along $\varphi$.

Proof. Let $N$ be a virtually small complex of $S$-modules, with witness $W$. Since $W$ is finitely built from $N$, it suffices to verify that it is virtually small over $R$. Then, $W$ being a small complex of $S$-modules, $\operatorname{fd}_{R} S$ finite implies $\operatorname{fd}_{R} W$ finite. For the same reason, $W$ is homologically finite over $S$. Replacing $N$ by $W$ allows one to apply Theorem 4.9, which gives the desired result.

Here is a direct corollary; confer Proposition 4.13 which asserts that, as an $R$ module, $\widehat{R}$ is not proxy small, unless $R$ is complete.

7.5 Corollary. Let $(R, \mathfrak{m}, k)$ be a local ring and $\widehat{R}$ its $\mathfrak{m}$-adic completion. Any virtually small complex of $\widehat{R}$-modules is virtually small over $R$.

In the preceding results the hypotheses have been on the $R$-module structure on $S$; the remaining ones focus on a property intrinsic to $S$. The theorem below implies that when $S$ is regular, any complex of $S$-modules whose homology is either finite 
or artinian, is proxy small over $R$; it extends [13, (3.2)]. We adopt the convention that a (not-necessarily-local) ring is regular if it is noetherian and has finite global dimension.

7.6 Theorem. Let $\varphi: R \rightarrow S$ be a homomorphism of rings with $R$ noetherian and the $R$-module $S$ finite. If $S$ is regular, then proxy smallness and virtual smallness descend along $\varphi$.

Proof. First consider the case when $\varphi$ is surjective. It suffices to verify that the $R$ module $S$ is proxy small; this is by Proposition 7.2. Let $I=\operatorname{Ker}(\varphi)$ and $K$ the Koszul complex on a finite generating set for $I$. Thanks to Proposition 4.4, it is enough to check that $K$ is finitely built from $S$, in $\mathscr{D}(R)$. For each integer $i$, the $R$-module $\mathrm{H}_{i}(K)$ is annihilated by $I$, and hence it is an $S$-module. Since $S$ is regular, it finitely builds $\mathrm{H}_{i}(K)$ over $S$, and hence also over $R$. Therefore, $K$ is finitely built from $S$ over $R$; see 3.10 .

Now we turn to the general case. Pick elements $s_{1}, \ldots, s_{e}$ in $S$ that generate $S$ as an $R$-module. Since $R$ is noetherian, for each integer $n$, the $R$-submodule of $S$ generated by the set $\left\{s_{n}, s_{n}^{2}, s_{n}^{3}, \ldots\right\}$ is finitely generated; that is to say, there exists a monic polynomial $f_{n}\left(x_{n}\right)$ in the ring $R\left[x_{n}\right]$ such that $f_{n}\left(s_{n}\right)=0$ in $S$. Set

$$
\widetilde{R}=R\left[x_{1}, \ldots, x_{e}\right] /\left(f_{1}\left(x_{1}\right), \ldots, f_{e}\left(x_{e}\right)\right) .
$$

The ring homomorphism $R\left[x_{1}, \ldots, x_{e}\right] \rightarrow S$ defined by $x_{n} \mapsto s_{n}$ is surjective and factors through $\widetilde{R}$, to yield a homomorphism $\pi: \widetilde{R} \rightarrow S$. Thus, one obtains a diagram $R \stackrel{\eta}{\longrightarrow} \widetilde{R} \stackrel{\pi}{\longrightarrow} S$ of homomorphisms of rings with $\pi \circ \eta=\varphi$, where $\eta$ is the canonical inclusion. Because $\pi$ is surjective, the already established part of the proposition yields that proxy smallness and virtual smallness descend along it. By Proposition 7.2, these properties descend also along $\eta$ because the $R$-module $\widetilde{R}$ is finite and free, as can be verified easily. These give the desired conclusion.

Once again, one has a better result for local homomorphisms.

7.7 Theorem. Let $\varphi:(R, \mathfrak{m}, k) \rightarrow(S, \mathfrak{n}, l)$ be a local homomorphism. If $S$ is regular, then virtual smallness descends along $\varphi$.

Proof. Proposition 4.5 says that the Koszul complex $K$ of $S$ serves as a witness for virtual smallness in $\mathscr{D}(S)$. Thus, for the desired result, it suffices to prove that $K$ is virtually small in $\mathscr{D}(R)$. The ring $S$ being regular, $K \simeq l$, so one needs to check that $l$ is virtually small in $\mathscr{D}(R)$. However, as a $k$-module $l$ finitely builds $k$; this is another way of saying that $k$ is a direct summand of $l$. Since the action of $R$ on $l$ factors through $k$, one deduces that $l$ finitely builds $k$ in $\mathscr{D}(R)$. It remains to recall that $k$ finitely builds the Koszul complex of $R$; see 4.7 . 


\section{Ascent}

Let $\psi: Q \rightarrow R$ be a local homomorphism. We say that virtual smallness ascends along $\psi$ if each complex of $R$-modules that is virtually small over $Q$ is also virtually small over $R$; the explanation for the choice of language is contained in the discussion at the beginning of Section 7 .

The main contribution of this section is Theorem 8.3 below that gives one criterion for ascent of the properties under consideration. Stating it requires that we enlarge our universe from rings to (commutative) Differential Graded algebras, and consider some notions from Section 3, introduced for rings, in this more general context. The reader may refer to [2] for basic results on DG objects in the commutative setting.

8.1. Let $A$ be a DG algebra. Akin to the case of rings, the derived category of DG $A$-modules is a triangulated category, which we denote $\mathcal{D}(A)$. Thus, one has the notion of one DG $A$-module being finitely built from another, and most basic remarks in Section 3, notably 3.9, 3.12, and 3.13, apply to DG $A$-modules. In particular, Definition 4.1 extends to DG $A$-modules.

In what follows, the homological algebra of the 'derived diagonal' homomorphism plays a crucial role. The next paragraph takes the first steps towards explaining this statement; see 8.10.

8.2. Let $\phi: Q[X] \stackrel{\simeq}{\longrightarrow} R$ be a DG algebra model of $\psi$; the construction of DG models is explained in $[2, \S(2.2)]$. In the sequel, we write $Q[X, X]$ for DG algebra $Q[X] \otimes_{Q} Q[X]$. As the graded algebra underlying $Q[X]$ is graded-commutative, the category of DG $Q[X]$-bimodules coincides with the category of DG (left) $Q[X, X]$ modules. In this identification, a DG $Q[X]$-bimodule $U$ acquires a structure of a DG $Q[X, X]$-module via the prescription

$$
\left(r_{1} \otimes r_{2}\right) \cdot u=(-1)^{\left|r_{2}\right||u|} r_{1} u r_{2}
$$

for $\left(r_{1} \otimes r_{2}\right) \in Q[X, X]$ and $u \in U$. This remark applies, in particular, to $Q[X]$ itself, with its canonical $Q[X]$-bimodule structure.

8.3 Theorem. Let $\psi: Q \rightarrow R$ be a homomorphism of rings and $Q[X]$ a DG model for $\psi$. Assume that $Q[X, X]$ is finitely built from $Q[X]$ in $\mathcal{D}(Q[X, X])$. The following statements hold.

(a) Virtual smallness and proxy smallness ascend along $\psi$.

(b) If $Q$ is noetherian and $R$ is module finite over $Q$, then the $Q$-module $R$ is small, and virtual smallness and proxy smallness also descend along $\psi$. 
The proof of the theorem above is given in 8.8. For now, we would like to make a few remarks on its formulation.

8.4 Remarks. Given that the category of $\mathrm{DG} \mathrm{Q}[\mathrm{X}, \mathrm{X}]$-modules is equivalent to that of DG $Q[X]$-bimodules, the ruling hypothesis in the theorem may stated as: $Q[X, X]$ is finitely built from $Q[X]$ as DG bimodules over $Q[X]$. Moreover, the hypothesis does not depend on the choice of the model for $\psi$; this statement is clarified in Remark 8.10. Therefore one may formulate the theorem above without taking recourse to any specific models; this is done in Remark 8.10.

Another noteworthy point is that there is nothing sacrosanct about commutative DG algebras: any suitable category of algebras that contains the commutative rings - for example, simplicial algebras - would do just as well. We have opted to work in that category for it suffices for the present need, and while the arguments we give do exploit the commutativity of the DG algebras, they can be modified to work in the desired generality; confer Remark 8.11.

One bonus of working with DG algebras rather than rings - which may be considered as DG algebras concentrated in degree zero - is that in testing whether one DG module is built from another one has the flexibility provided by the next result.

8.5 Proposition. Consider a morphism $\phi: A \rightarrow A^{\prime}$ of $D G$ algebras, $D G$ A-modules $U, V$, and $D G A^{\prime}$-modules $U^{\prime}, V^{\prime}$. Let $f: U \rightarrow U^{\prime}$ and $g: V \rightarrow V^{\prime}$ be $\phi$-linear morphisms of $D G$ modules.

When $\mathrm{H}(\phi), \mathrm{H}(f)$, and $\mathrm{H}(g)$ are bijective, the $D G A$-module $V$ is finitely built from $U$ if and only if the $D G A^{\prime}$-module $V^{\prime}$ is finitely built from $U^{\prime}$.

Proof. To begin with note that in $\mathscr{D}(A)$ the morphism $f$ factors as

$$
U=A \otimes_{A}^{\mathbf{L}} U \stackrel{\phi \otimes_{A}^{\mathbf{L}} U}{\longrightarrow} A^{\prime} \otimes_{A}^{\mathbf{L}} U \stackrel{A^{\prime} \otimes_{\phi}^{\mathbf{L}} f}{\longrightarrow} A^{\prime} \otimes_{A^{\prime}}^{\mathbf{L}} U^{\prime}=U^{\prime}
$$

where $A^{\prime} \otimes_{\phi}^{\mathbf{L}} f$ is a morphism of DG $A^{\prime}$-modules. Since $\mathrm{H}(\phi)$ is bijective, so is $\mathrm{H}\left(\phi \otimes_{A}^{\mathbf{L}} U\right)$, hence the bijectivity of $\mathrm{H}(f)$ implies that of $\mathrm{H}\left(A^{\prime} \otimes_{\phi}^{\mathbf{L}} f\right)$. By the same token, $\mathrm{H}\left(A^{\prime} \otimes_{\phi}^{\mathbf{L}} g\right)$ is bijective. Now, when the DG $A$-module $V$ is finitely built from $U$, the DG algebra version of 3.12 yields that the DG $A^{\prime}$-module $A^{\prime} \otimes_{A}^{\mathbf{L}} V$ is finitely built from $A^{\prime} \otimes{ }_{A}^{\mathbf{L}} U$. Thus, by the preceding discussion, we deduce that $V^{\prime}$ is finitely built from $U^{\prime}$, as desired. The converse is immediate from 3.13.

One useful consequence of the preceding result is the

8.6 Corollary. Let $\phi: A \rightarrow A^{\prime}$ be a morphism of $D G$ algebras. If $\mathrm{H}(\phi)$ is bijective, then virtual smallness and proxy smallness ascend and descend along $\phi$. 
Proof. We deal with virtual smallness, and leave it to the reader to worry about proxy smallness. Let $U^{\prime}$ be a DG $A^{\prime}$-module.

In $\mathscr{D}(A)$, every $A^{\prime}$-linear morphism is also $A$-linear and $A \simeq A^{\prime}$. Thus, if $U^{\prime}$ is virtually small over $A^{\prime}$, then it is virtually small over $A$.

Conversely, if $U^{\prime}$ is virtually small over $A$, with witness $V$, then $V^{\prime}=A^{\prime} \otimes{ }_{A}^{\mathbf{L}} V$, viewed as a DG $A^{\prime}$-module via the left hand factor of the (derived) tensor product, is a witness that $U^{\prime}$ is virtually small over $A^{\prime}$. Indeed, $\phi$ being an quasiisomorphism, so is the $\phi$-linear morphism

$$
\phi \otimes_{A}^{\mathbf{L}} V: V=A \otimes_{A}^{\mathbf{L}} V \rightarrow A^{\prime} \otimes_{A}^{\mathbf{L}} V=V^{\prime} .
$$

Set $g=\phi \otimes{ }_{A}^{\mathbf{L}} V$. Now, Proposition 8.5 applied with $f=\phi$ yields that in $\mathscr{D}\left(A^{\prime}\right)$, the DG-module $V^{\prime}$ is finitely built from $A^{\prime}$, and applied with $f=\mathrm{id}^{U^{\prime}}$ yields that $V^{\prime}$ is finitely built from $U^{\prime}$. This is the desired conclusion.

The proof of the Theorem 8.3 uses a well-known adjunction isomorphism:

8.7. If $U$ and $V$ are DG $Q[X]$-modules, then in $\mathscr{D}(Q[X])$ one has

$$
\mathbf{R H o m}_{Q[X, X]}\left(Q[X], \mathbf{R H o m}_{Q}(U, V)\right) \simeq \mathbf{R}_{H^{\prime}} \operatorname{Hom}_{Q[X]}(U, V) .
$$

Indeed, $Q[X]$ being graded-commutative, $\operatorname{Hom}_{Q}(U, V)$ is a DG $Q[X, X]$-module, with

$$
\left[\left(r_{1} \otimes Q r_{2}\right) \cdot f\right](u)=(-1)^{\left|r_{2}\right||f|} r_{1} f\left(r_{2} u\right) .
$$

By MacLane [23, Chapter VI, (8.6)], there is an isomorphism of DG $Q[X]$-modules

$$
\operatorname{Hom}_{Q[X, X]}\left(Q[X], \operatorname{Hom}_{Q}(U, V)\right) \cong \operatorname{Hom}_{Q[X]}(U, V) ;
$$

the derived version of this isomorphism is the one we seek.

We are now ready for the

8.8. Proof of Theorem 8.3. (a) We focus on virtual smallness; the argument for proxy smallness is similar. Recall that $\phi: Q[X] \rightarrow R$ is a DG algebra model for $\psi$. Let $\eta: Q \rightarrow Q[X]$ be the canonical inclusion; one has $\phi \circ \eta=\psi$. Thanks to Corollary 8.6, since $\phi$ is a quasiisomorphism, it suffices to prove that virtual smallness ascends along $\eta$.

To this end, let $U$ be a DG $Q[X]$-module which is virtually small over $Q$, and let $W$ be a witness that this is so. We claim that $Q[X] \otimes_{Q}^{\mathbf{L}} W$, viewed as a DG $Q[X]$ module via the left hand factor of the tensor product, is a witness that $U$ is virtually small over $Q[X]$. Indeed, the complex $Q[X] \otimes{ }_{Q}^{\mathbf{L}} W$ is finitely built from $Q[X]$ since 
$W$ is finitely built from $Q$. The argument that it is also finitely built from $U$ is contained in the diagram:

$$
U=Q[X] \otimes_{Q[X]}^{\mathbf{L}} U \underset{\overline{Q[X]}}{\Longrightarrow} Q[X, X] \otimes_{Q[X]}^{\mathbf{L}} U \simeq Q[X] \otimes_{Q}^{\mathbf{L}} U \underset{\overline{Q[X]}}{\Longrightarrow} Q[X] \otimes_{Q}^{\mathbf{L}} W
$$

where the equivalence is evident, once we recall that $Q[X, X]=Q[X] \otimes_{Q} Q[X]$, and the second implication is given by 3.12, since $W$ is finitely built from $U$.

The justification for the first implication is more delicate: as noted before, the category of DG $Q[X, X]$-modules is the same as the category of DG $Q[X]$-bimodules. Let $V$ be a DG $Q[X, X]$-module. The derived tensor product $V \otimes_{Q[X]}^{\mathbf{L}} U$, whose construction uses the DG right $Q[X]$-module structure of $V$, inherits from $V$ the structure of a DG left $Q[X]$-module. Moreover, the assignment $V \mapsto V \otimes_{Q[X]}^{\mathbf{L}} U$ is an exact functor from $\mathscr{D}(Q[X, X])$ to $\mathscr{D}(Q[X])$. Thus, if $V$ is finitely built from $V^{\prime}$ as DG $Q[X, X]$-modules, then $V \otimes_{Q[X]}^{\mathbf{L}} U$ is finitely built from $V^{\prime} \otimes_{Q[X]}^{\mathbf{L}} U$ as DG $Q[X]$-modules. The special case $V=Q[X, X]$ and $V^{\prime}=Q[X]$ of the last statement is the desired implication.

This completes the proof that virtual smallness ascends along $\psi$.

(b) It is enough to prove that $R$ is small over $Q$ : the descent of virtual smallness and proxy smallness follow from Proposition 7.2. As to that, thanks to Proposition 2.7, it suffices to verify that, homologically, $\operatorname{RHom}_{Q}(R, N)$ is bounded below for each $Q$-module $N \neq 0$. Since $Q[X] \simeq R$ in $\mathscr{D}(Q)$, this is tantamount to verifying that

$$
\inf \left(\operatorname{RHom}_{Q}(Q[X], N)\right)>-\infty .
$$

To this end, note that in $\mathscr{D}(Q[X])$ one has isomorphisms

$$
\mathbf{R H o m}_{Q[X, X]}\left(Q[X], \mathbf{R H o m}_{Q}(Q[X], N)\right) \simeq \operatorname{RHom}_{Q[X]}(Q[X], N) \simeq N
$$

where the one on the left is given by 8.7, while that on the right needs no explanation. In particular, the infimum of the DG module on the left is zero. From this remark, our hypothesis that $Q[X, X]$ is finitely built from $Q[X]$ in $\mathscr{D}(Q[X, X])$, and the isomorphism

$$
\mathbf{R H o m}_{Q}(Q[X], N) \simeq \mathbf{R H o m}_{Q[X, X]}\left(Q[X, X], \mathbf{R H o m}_{Q}(Q[X], N)\right)
$$

we deduce: either $\mathbf{R H o m}_{Q}(Q[X], N)$ is zero, or its infimum is finite.

The remainder of this section expatiates on the remarks following Theorem 8.3.

8.9. Let $Q[Y]$ be another DG algebra model of $\psi$. There is then a morphism of DG $Q$-algebras $\kappa: Q[X] \rightarrow Q[Y]$ such that

(i) $\kappa$ is a quasiisomorphism;

(ii) $\kappa \otimes_{Q} \kappa: Q[X, X] \rightarrow Q[Y, Y]$ is a quasiisomorphism; 
(iii) $\kappa$ is $\left(\kappa \otimes_{Q} \kappa\right)$-linear.

Indeed, the lifting property of models [2, (2.1.9)] yields a commutative diagram of morphisms of DG $Q$-algebras

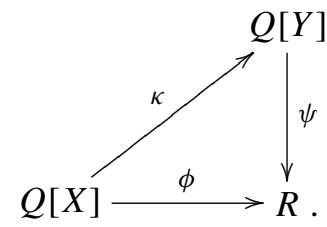

Since $\phi$ and $\psi$ are both quasiisomorphisms, so is $\kappa$. Therefore, $\kappa \otimes_{Q} \kappa$ is also a quasiisomorphism, because the DG $Q$-modules $Q[X]$ and $Q[Y]$ are semifree; see [2, (1.3.3)]. Finally, it is elementary to verify that $\kappa$ is $\left(\kappa \otimes_{Q} \kappa\right)$-linear.

8.10 Remarks. In what follows, when we speak about the DG $Q$-algebra $R \otimes_{Q}^{\mathbf{L}} R$, we mean the DG $Q$-algebra $Q[X, X]$, and when we speak of $R$ as a DG $\left(R \otimes_{Q}^{\mathbf{L}} R\right)$ module, we mean $Q[X]$ viewed as a DG $Q[X, X]$-module. Remark 8.9 explains the sense in which these are independent of the choice of the DG model for $\psi$. In addition, it is immediate from Proposition 8.5 and 8.9 that condition that $R \otimes_{Q}^{\mathbf{L}} R$ is finitely built from $R$ as $\left(R \otimes_{O}^{\mathbf{L}} R\right)$-modules is also intrinsic to $\psi$. The upshot is that the hypothesis of Theorem 8.3 admits a rather more pleasing formulation.

Theorem. Let $\psi: Q \rightarrow R$ be a homomorphism of rings. Assume that $R \otimes{ }_{Q}^{\mathbf{L}} R$ is finitely built from $R$ in $\mathscr{D}\left(R \otimes{ }_{Q}^{\mathbf{L}} R\right)$. The following statements hold.

(a) Virtual smallness and proxy smallness ascend along $\psi$.

(b) If $Q$ is noetherian and $R$ is module finite over $Q$, then the $Q$-module $R$ is small, and virtual smallness and proxy smallness also descend along $\psi$.

The last item in this section is a more category theoretic proof of the following extension of Theorem 8.3 (b). The argument given may be more illuminating than the one given above; besides, it lends itself to use in other contexts.

8.11 Proposition. Let $\psi: Q \rightarrow R$ be a homomorphism of rings and set $R^{e}=R \otimes{ }_{Q}^{\mathbf{L}} R$. If $R$ is proxy small over $R^{e}$, with witness $W$, then $V=R \otimes R_{R^{e}} W$, viewed as a complex of $R$-modules via the right hand factor, has the following properties:

(i) In $\mathscr{D}(Q)$, the complex $V$ is finitely built from $Q$.

(ii) In $\mathscr{D}(R)$, the complex $V$ is finitely built from $R$.

(iii) In $\mathscr{D}(R)$, the complex $R$ is built from $V$.

In particular, as a $Q$-module, $R$ is proxy small, with witness $V$. 
Proof. Property (ii) holds by base change, since $W$ is finitely built from $R$, in $R^{e}$. Similarly, since $R$ is built from $W$ in $\mathscr{D}\left(R^{e}\right)$, one obtains that $R^{e}$ is built from $V$ in $\mathscr{D}(R)$. However $R$ itself is built from $R^{e}$, where we view the latter as an $R$-module via the left hand factor. Thus, $R$ is built from $V$ over $R$, which settles (iii).

For (i), it suffices to prove - see 3.7 - that, given complexes of $Q$-modules $\left\{M_{\lambda}\right\}_{\lambda \in \Lambda}$, indexed by a set $\Lambda$, the canonical map

$$
\eta: \amalg_{\lambda \in \Lambda} \mathbf{R H o m}_{Q}\left(V, M_{\lambda}\right) \rightarrow \mathbf{R H o m}_{Q}\left(V, \amalg_{\lambda \in \Lambda} M_{\lambda}\right)
$$

is an isomorphism. For each DG $R^{e}$-module $X$, denote $\eta(X)$ the morphism

$$
\amalg_{\lambda \in \Lambda} \operatorname{RHom}_{R^{e}}\left(X, \mathbf{R H o m}_{Q}\left(R, M_{\lambda}\right)\right) \rightarrow \mathbf{R H o m}_{R^{e}}\left(X, \mathbf{R H o m}_{Q}\left(R, \amalg_{\lambda \in \Lambda} M_{\lambda}\right)\right)
$$

induced by $\eta$. Observe that $\eta(R)$ is an isomorphism; this is a consequence of the isomorphism in 8.7. Thus, $\eta(X)$ is an isomorphism whenever $X$ is finitely built from $R$; in particular $\eta(W)$ is an isomorphism. However

$$
\mathbf{R H o m}_{R^{e}}\left(W, \mathbf{R H o m}_{Q}(R,-)\right) \simeq \mathbf{R H o m}_{Q}\left(R \otimes_{R^{e}}^{\mathbf{L}} W,-\right)
$$

by adjunction, therefore $\eta(W)=\eta$, and hence $\eta$ is an isomorphism, as desired.

\section{Complete intersection local rings}

The results described in this section are all inspired by the question: what rings have the property that every homologically finite complex over them is virtually small? One of our main conclusions is contained in Theorem 9.4; the following result leads up to it.

9.1 Theorem. Let $\psi: Q \rightarrow R$ be an surjective homomorphism of noetherian rings. If $\operatorname{Ker}(\psi)$ can be generated by a regular sequence, then virtual smallness and proxy smallness ascend and descend along $\psi$.

Proof. Let $r_{1}, \ldots, r_{c}$ be a regular sequence generating the ideal $\operatorname{Ker}(\psi)$, and set $Q_{i}=Q /\left(r_{1}, \ldots, r_{i}\right)$. Consider canonical surjections

$$
Q_{0}=Q \stackrel{\psi_{1}}{\longrightarrow} Q_{1} \stackrel{\psi_{2}}{\longrightarrow} \cdots \stackrel{\psi_{c-1}}{\longrightarrow} Q_{c-1} \stackrel{\psi_{c}}{\longrightarrow} Q_{c}=R .
$$

Evidently, it suffices to verify that virtual smallness and proxy smallness ascend and descend along each of the $\psi_{i}$. Note that $\operatorname{Ker}\left(\psi_{i}\right)$ is generated by the regular element $r_{i}$ in $Q_{i-1}$. Thus, we may assume that $R=Q /(r)$, with $r$ a regular element.

The Koszul complex on $r$ is the DG algebra $Q[x \mid \partial(x)=r]$, with $x$ an (exterior) variable in degree 1 . The complex of $Q$-modules underlying it is

$$
0 \rightarrow Q \stackrel{r}{\longrightarrow} Q \rightarrow 0 .
$$


Consider the canonical surjection $\phi: Q[x] \rightarrow R$ of DG $Q$-algebra. Since $r$ is a regular element, $\mathrm{H}_{1}(Q[x])=0$, and so $\phi$ is a quasiisomorphism; that is to say, $Q[x]$ is a DG algebra model for $\psi$.

As a complex of $Q$-modules, $Q[x]$, and hence $R$, is small, so the assertion on descent is contained in Proposition 7.2.

To prove ascent, we verify the hypotheses of Theorem 8.3. This entails verifying that $Q[x, x]$ is finitely built from $Q[x]$ in $\mathscr{D}(Q[x, x])$. A direct computation reveals that the $Q[x, x]$-module $Q[x]$ is cyclic, and its annihilator is the DG ideal generated by the element $(x \otimes 1-1 \otimes x)$, which we denote $\Delta$. Moreover, the annihilator in $Q[x, x]$ of the element $\Delta$ is the ideal $(\Delta)$. Consequently, one has an exact sequence of DG $Q[x, x]$-modules

$$
0 \rightarrow Q[x] \stackrel{\eta}{\longrightarrow} Q[x, x] \stackrel{\epsilon}{\longrightarrow} Q[x] \rightarrow 0
$$

where $\eta(1)=\Delta$ and $\epsilon$ is the canonical surjection. Thus, as DG $Q[x, x]$-modules, $Q[x, x]$ is finitely built from $Q[x]$.

9.2 Remark. Abstracting the crux of the preceding proof one obtains:

Let $A$ be a graded-commutative DG algebra and $\phi: A \rightarrow A[X]$ a semi-free extension, where the set $X$ is finite and concentrated in odd degrees. Then virtually smallness and proxy smallness ascend and descend along $\phi$.

One benefit of this version of the result is that it contains the extension of Theorem 9.1 where one assumes only that the Koszul complex on a minimal generating set for $\operatorname{Ker}(\psi)$ is acyclic.

Now we apply the preceding theorem to complete intersection. First, a recap.

9.3. Let $(R, \mathfrak{m}, k)$ be a local ring and denote $\widehat{R}$ its $\mathfrak{m}$-adic completion. A Cohen presentation of $\widehat{R}$ is a surjective local homomorphism $Q \rightarrow R$ with the ring $Q$ regular; one such always exists, by Cohen's Structure Theorem.

The ring $R$ is complete intersection if the kernel of some, equivalently, any, Cohen presentation $Q \rightarrow \widehat{R}$ is generated by a regular sequence. In this case, if $\pi: Q \rightarrow R$ is a surjective homomorphism with $Q$ a regular local ring, then $\operatorname{Ker}(\pi)$ is generated by a regular sequence; see $[8, \S(2.3)]$ for details.

Here is the advertized result on complete intersection. As to its statement, we should like to remark that no examples are known of complete intersections that are not quotients of regular rings.

9.4 Theorem. Let $(R, \mathfrak{m}, k)$ be a complete intersection local ring, and $M$ a complex of $R$-modules.

If $\mathfrak{m} \in \operatorname{Supp} M$, then $M$ is virtually small. If $R$ is a quotient of a regular local ring and $\mathrm{H}(M)$ is either finite or artinian, then $M$ is proxy small. 
Proof. When $R$ is a quotient of a regular local ring say $Q$, the kernel of the canonical surjection $Q \rightarrow R$ is generated by a regular sequence; this was noted in 9.3. The local ring $Q$ being regular, when $\mathrm{H}(M)$ is either finite or artinian, Corollary 4.10 yields that $M$ is proxy small over $Q$; given this, Theorem 9.1 implies $M$ is proxy small over $R$.

Now we tackle the general case; in doing so, we may as well assume $\mathrm{H}(M) \neq 0$. Let $K$ be the Koszul complex of $R$. Since $K$ is finitely built from $R$, the complex $K \otimes_{R} M$ is finitely built from $M$. Moreover, using 3.16 one obtains

$$
\operatorname{supp}\left(K \otimes_{R}^{\mathbf{L}} M\right)=\operatorname{supp} K \cap \operatorname{supp} M=\{\mathfrak{m}\} \cap \operatorname{supp} M .
$$

Thus, our hypothesis ensures that $\operatorname{supp}\left(K \otimes_{R}^{\mathbf{L}} M\right)=\{\mathfrak{m}\}$, and in particular, that $\mathrm{H}\left(K \otimes_{R}^{\mathbf{L}} M\right) \neq 0$. Hence, it suffices to prove that $K \otimes_{R} M$ is virtually small, and so, replacing $M$ by this complex, one may assume $\operatorname{supp} M=\{\mathfrak{m}\}$, and $\mathfrak{m} \mathrm{H}(M)=0$, see 2.9 (a), that is to say, $\mathrm{H}(M)$ is a $k$-vector space.

Let $\widehat{R}$ be the $\mathfrak{m}$-adic completion of $R$. Now that $\mathfrak{m} \mathrm{H}(M)=0$, the canonical homomorphism $M \rightarrow \widehat{R} \otimes_{R} M$ of complexes of $R$-modules is a quasiisomorphism. Thus, $\mathrm{H}\left(\widehat{R} \otimes_{R} M\right)$ is again a $k$-vector space and $\mathfrak{m} \widehat{R}$, the maximal ideal of $\widehat{R}$, is contained in $\operatorname{supp}_{\widehat{R}}\left(\widehat{R} \otimes_{R} M\right)$. Cohen's structure theorem gives a surjective local homomorphism $Q \rightarrow \widehat{R}$ with $Q$ regular; its kernel is generated by a regular sequence, since $R$ is complete intersection. Proposition 4.11 implies that $\widehat{R} \otimes_{R} M$ is virtually small over $Q$, and then Theorem 9.1 implies that $\widehat{R} \otimes_{R} M$ is virtually small over $\widehat{R}$. Applying Corollary 7.5 one obtains that $\widehat{R} \otimes_{R} M$ is virtually small over $R$. It remains to recall that $M \simeq \widehat{R} \otimes_{R} M$.

9.5 Remark. The class of virtually small complexes identified by the preceding result is by no means exhaustive. For instance, one can construct many more of those via Proposition 4.16, or by mimicking Example 4.3.

The theorem above and Corollary 4.10 suggest the following considerations.

9.6 Remarks. A homologically finite complex $M$ of $R$-modules is said have finite CI-dimension if there is a diagram of local homomorphisms $R \rightarrow R^{\prime} \leftarrow Q$ such that

(i) $R \rightarrow R^{\prime}$ is flat;

(ii) $Q \rightarrow R^{\prime}$ is surjective, with kernel generated by a regular sequence, and

(iii) $\mathrm{fd}_{Q}\left(R^{\prime} \otimes_{R} M\right)$ is finite.

Small complexes have this property, as do homologically finite complexes over complete intersections; see Avramov, Gasharov, and Peeva [6] for details. The question naturally arises: is a complex of finite CI-dimension virtually small?

Assume $M$ is a complex of finite CI-dimension. Since the complex $R^{\prime} \otimes_{R} M$ is homologically finite over $R^{\prime}$, and hence also over $Q$, condition (iii) implies that it is 
small over $Q$. Thus, condition (ii) and Theorem 9.1 yield that $R^{\prime} \otimes_{R} M$ is virtually small over $R^{\prime}$. Thus, what remains before one can conclude that $M$ is virtually small, is to deal with the

Question. Let $M$ and $N$ be homologically finite complexes of $R$-modules.

$$
\text { Does }\left(M \otimes_{R}^{\mathbf{L}} R^{\prime}\right) \underset{R^{\prime}}{\Longrightarrow}\left(N \otimes_{R}^{\mathbf{L}} R^{\prime}\right) \text { imply } M \underset{R}{\Longrightarrow} N \text { ? }
$$

In words: if $N \otimes_{R}^{\mathbf{L}} R^{\prime}$ is finitely built from $M \otimes_{R}^{\mathbf{L}} R^{\prime}$, then is $N$ finitely built from $M$ ?

Theorem 9.4 allows one to specialize many results in Section 5 to the case where $R$ is complete intersection; these throw new light on homological algebra over such rings. We spell out the one corresponding to Theorem 5.10 because it identifies new scenarios when the complete intersection property descends along a local homomorphism.

9.7 Theorem. Let $\psi:(Q, \mathfrak{n}, l) \rightarrow(R, \mathfrak{m}, k)$ be a local homomorphism and $M$ a homologically finite complex of $R$-modules with $\mathrm{H}(M) \neq 0$. If $R$ is complete intersection, then

$$
\operatorname{cx}_{Q} h \leq \operatorname{codim} R+\operatorname{cx}_{\psi} M \text { and } \operatorname{curv}_{Q} h \leq \max \left\{1, \operatorname{curv}_{\psi} M\right\} .
$$

In particular, if $\operatorname{curv}_{\psi} M \leq 1$, then $Q$ is complete intersection. If $\mathrm{fd}_{Q} M$ is finite, then $Q$ is complete intersection and $\operatorname{codim} Q \leq \operatorname{codim} R$.

9.8 Remark. The theorem above is a common generalization of Parts (1) and (2) of [7, (13.1)], which in turn contain the descent of the complete intersection property along flat homomorphisms.

For use in the proof of the theorem, we recall a characterization of complete intersection rings in terms of asymptotic invariants of $k$.

9.9. Let $(R, \mathfrak{m}, k)$ be a local ring. The following conditions are equivalent:

(i) $R$ is complete intersection;

(ii) $\mathrm{cx}_{R} k=\operatorname{codim} R$;

(iii) $\operatorname{curv}_{R} k \leq 1$.

The proof that (i) $\Longrightarrow$ (ii) is contained in a result of Tate, while the reverse implication is due to Gulliksen. The remaining non-trivial implication, (iii) $\Longrightarrow$ (ii) was established by Avramov. The reader may refer to $[2, \S(8.1)]$ for details. 
Proof of Theorem 9.7. From Theorem 9.4 one obtains that $M$ is virtually small; with this on hand, Theorem 5.10 with $Y$ specialized to $k$ yields

$$
\operatorname{cx}_{\psi} k \leq \operatorname{cx}_{R} k+\mathrm{cx}_{\psi} M \text { and } \operatorname{curv}_{\psi} k \leq \max \left\{\operatorname{curv}_{R} k, \operatorname{curv}_{\psi} M\right\} .
$$

It can be deduced easily from [7, (5.1.2)] that $\mathrm{cx}_{\psi} k=\mathrm{cx}_{Q} h$ and $\operatorname{curv}_{\psi} k=\operatorname{curv}_{Q} h$; these, in conjunction with the inequalities above, and characterizations of complete intersection in 9.9, yield the inequalities we seek.

If $\operatorname{curv}_{\psi} M \leq 1$, then the inequality $\operatorname{curv}_{Q} h \leq \max \left\{1, \operatorname{curv}_{\psi} M\right\}$, which has been verified, implies curv $Q h \leq 1$, so $Q$ is complete intersection, by 9.9.

Suppose $\operatorname{fd}_{Q} M$ is finite. Then $\operatorname{cx}_{\psi} M=0=\operatorname{curv}_{\psi} M$, by [7, (7.1.3.1)], so the inequalities that have been established yield $\operatorname{cx}_{Q} h \leq \operatorname{cx}_{R} k$ and $\operatorname{curv}_{Q} h \leq 1$. Now we again invoke 9.9 to deduce from the latter inequality that $Q$ is complete intersection, and hence from the former that $\operatorname{codim} Q \leq \operatorname{codim} R$.

In the remainder of this article we focus on the problem below, which seeks a converse to Theorem 9.4. A positive answer would provide, in conjunction with Theorem 9.4, a new homotopical characterization of local complete intersections.

9.10 Question. Over a local ring $R$, if each homologically finite complex is virtually small, then is $R$ complete intersection?

The next result takes us a part of the way toward an affirmative answer.

9.11 Theorem. Let $R$ be a local ring. If each homologically finite complex of $R$ modules is virtually small, then $R$ is Gorenstein.

Proof. Theorem 5.3 yields the desired conclusion, once we observe that $R$ has a homologically finite complex of finite injective dimension. To verify the latter claim, let $K$ be the Koszul complex of $R$ and $E$ the injective hull of the residue field $k$ of $R$. The complex $M=\operatorname{Hom}_{R}(K, E)$ has the desired properties. Indeed, $\mathrm{H}(M)$ is non-zero and artinian, for example, by Matlis duality, and the maximal ideal of $R$ annihilates it. Thus $\mathrm{H}(M)$ is a non-zero $k$-vector space of finite rank, and hence a finite $R$-module. Since $E$ is injective and $K$ is a finite free complex, $\operatorname{id}_{R} M$ is finite, as desired.

One may also deduce the preceding result from a version for homomorphisms, described below; however, the direct proof is easier. We note that a Gorenstein homomorphism is one which is quasi-Gorenstein and has finite flat dimension.

9.12 Theorem. Let $\psi: Q \rightarrow R$ be a module finite local homomorphism with $\mathrm{fd}_{Q} R$ finite. If each homologically finite complex of $R$-modules that is small over $Q$ is virtually small over $R$, then the homomorphism $\psi$ is Gorenstein. 
Proof. Set $D=\mathbf{R H o m}_{Q}(R, Q)$; via $R$, this acquires a structure of a complex of $R$-modules. Since $\psi$ is module finite, $D$ is a dualizing complex for $\psi$, by [4, (5.12)]. Viewed as a complex of $Q$-modules, $R$ is small, by 3.8 , and hence so is $D$. The hypothesis now entails $D$ is virtually small over $R$. Now invoke Theorem 6.7.

The converse to Theorem 9.11 does not hold; in that, there are Gorenstein rings having complexes, and even modules, that are homologically finite but not virtually small. One family of examples is described below.

9.13 Example. Let $Q$ be a Cohen-Macaulay local ring with dualizing module $\omega$. Set $R=Q \ltimes \omega$, the trivial extension of $Q$ by $\omega$, and view $Q$ as an $R$-module via the canonical surjection $R \rightarrow Q$. The local ring $R$ is Gorenstein; see [8, (3.3.6)]. However, if $Q$ is virtually small as an $R$-module, then $Q$ is Gorenstein.

Indeed, if the $R$-module $Q$ is virtually small, then Corollary 5.7 implies that $\mathrm{fd}_{Q} R$ is finite; thus $\mathrm{fd}_{Q} \omega$ is finite, so $Q$ is Gorenstein; see [11, (3.4.12)], or use (c) $\Longrightarrow$ (a) of Theorem 5.3, keeping in mind that $\operatorname{id}_{Q} \omega$ is finite.

Now we describe another class of Gorenstein rings having modules that are not virtually small. The special case $\operatorname{rank}_{k} V=3$ was the first ring recognized, by Macaulay it seems, to be Gorenstein but not complete intersection; this is one reason for our interest in this example.

9.14 Example. Let $k$ be a field of characteristic $\neq 2$ and $V$ a $k$-vector space of finite rank. Each symmetric bilinear form $\boldsymbol{q}$ on $V$ gives rise to a commutative, graded $k$-algebra

$$
R(\boldsymbol{q})=k \oplus V \oplus k \quad \text { with } v \cdot w=q(v, w) \text { for } v, w \text { in } V .
$$

This $k$-algebra is concentrated in degrees 0,1 and 2, is artinian and local.

Claim. Assume that the form $\boldsymbol{q}$ is non-degenerate. The following statements hold:

(a) $R(\boldsymbol{q})$ is Gorenstein; it is complete intersection if and only if $\operatorname{rank}_{k} V \leq 2$.

(b) When $\operatorname{rank}_{k} V \geq 3$, the $\operatorname{ring} R(\boldsymbol{q})$ has a module that is not virtually small.

Property (a) is well-known, and one justification for it is given below. To start with, it is convenient to set $d=\operatorname{rank}_{k} V$, and abbreviate $R(\boldsymbol{q})$ to $R$. The claims are all easy to verify when $d=1$, for then $R \cong k[x] /\left(x^{3}\right)$. Assume henceforth that $d \geq 2$; choosing an orthogonal basis for $V$ allows one to realize $R$ as

$$
R=\frac{k\left[x_{1}, \cdots, x_{d}\right]}{J} \text { where } J=\left(\left\{x_{1}^{2}-c_{i} x_{i}^{2}\right\}_{2 \leqslant i \leqslant d},\left\{x_{i} x_{j}\right\}_{1 \leqslant i<j \leq d}\right)
$$

where the $c_{i}$ are non-zero elements in $k$. Note that the prescribed generating set for $J$ is minimal, and that it contains $\left(d^{2}+d-2\right) / 2$ elements. 
Proof of (a). As $\boldsymbol{q}$ is non-degenerate, the socle of $R$ is precisely its degree two component, and hence of rank one. Since $R$ is artinian, one obtains that it is Gorenstein, by $[8,(3.2 .10)]$. Also, $R$ is complete intersection precisely when the number of generators of $J$, that is to say, $\left(d^{2}+d-2\right) / 2$, equals $d$. This happens if and only if $d=2$.

Proof of (b). We claim that the $R$-module below is not virtually small:

$$
M=R /\left(x_{1} x_{2}, x_{1}+c_{2} x_{2}, x_{3}, \ldots, x_{d}\right) .
$$

To see this, set $Q=k\left[x_{1}, \ldots, x_{d}\right] /\left(x_{1} x_{2}\right)$ and let $\psi: Q \rightarrow R$ be the canonical surjection. By Theorem 5.5, it suffices to prove that $\mathrm{fd}_{Q} M$ is finite, while $\mathrm{fd}_{Q} R$ is not. The first claim is easily settled: the sequence $x_{1}+c_{2} x_{2}, x_{3}, \ldots, x_{d}$ is regular on $Q$, so the Koszul complex on it is a minimal resolution of $M$ over $Q$. The nonfiniteness of $\operatorname{fd}_{Q} R$ is the special case $I=\left(x_{1} x_{2}\right)$ of the more general statement:

Claim. Let $I \subset J$ be a homogeneous ideal of $k\left[x_{1}, \ldots, x_{d}\right]$; set $Q=k\left[x_{1}, \ldots, x_{d}\right] / I$. If $I$ is generated by a regular sequence, then $\mathrm{fd}_{Q} R=\infty$.

Indeed, suppose $n=\mathrm{fd}_{Q} R$ is finite; then $n=\operatorname{dim} Q$, but this is not important for what follows. The homogeneous minimal $Q$-free resolution of $R$ reads

$$
0 \rightarrow \bigoplus_{j \in \mathbb{Z}} Q(-j)^{b_{n j}} \rightarrow \cdots \rightarrow \bigoplus_{j \in \mathbb{Z}} Q(-j)^{b_{1 j}} \rightarrow Q \rightarrow 0
$$

where the $b_{i j}$ are non-negative integers, and for each $i$, one has $b_{i j}=0$ for all but finitely many $j$. From the resolution above, and the additivity of Hilbert series on short exact sequences, one obtains an equality of formal power series

$$
\operatorname{Hilb}_{R}(s)=\operatorname{Hilb}_{Q}(s) \cdot\left(\sum_{i, j}(-1)^{i} b_{i j} s^{j}\right) .
$$

Let $r_{1}, \ldots, r_{c}$ be a regular sequence generating $I$ and set $d_{i}=\operatorname{deg} r_{i}$; note that $d_{i} \geq 2$, since $I \subset J$. Then

$$
\operatorname{Hilb}_{R}(s)=1+d s+s^{2} \quad \text { and } \quad \operatorname{Hilb}_{Q}(s)=\frac{\prod_{i=1}^{c}\left(1-t^{d_{i}}\right)}{(1-t)^{d}} .
$$

These formulas combined with $(\dagger)$ imply that the primitive $d_{1}$ th root of unity is a root of $1+d s+s^{2}$. This cannot happen because $d \geq 3$.

Acknowledgments. It is a pleasure to thank Lucho Avramov for numerous discussions on this work, and Sean Sather-Wagstaff for comments on this manuscript. Part of this research was done at the Mathematical Sciences Research Institute, Berkeley, where the authors met in February 2003 during the special year in commutative algebra. We are grateful to the MSRI for its hospitality. The second author thanks the University of Nebraska, Lincoln, for hospitality during a visit in November 2003. 
Vol. 81 (2006)

\section{References}

[1] D. Apassov, Almost finite modules. Comm. Algebra 27 (1999), 919-931. Zbl 0922.13012 MR 1672015

[2] L. L. Avramov, Infinite free resolutions. In Six Lectures on Commutative Algebra (Bellaterra, 1996), Progr. Math. 166, Birkhäuser, Basel 1998, 1-118. Zbl 0934.13008 MR 1648664

[3] L. Avramov, H.-B. Foxby, Homological dimensions of unbounded complexes. J. Pure. Appl. Algebra 71 (1991), 129-155. Zbl 0737.16002 MR 1117631

[4] L. Avramov, H.-B. Foxby, Ring homomorphisms and finite Gorenstein dimensions. Proc. London Math. Soc. (3) 75 (1997), 241-270. Zbl 0901.13011 MR 1455856

[5] L. Avramov, H.-B. Foxby, B. Herzog, Structure of local homomorphisms. J. Algebra 164 (1994), 124-145. Zbl 0798.13002 MR 1268330

[6] L. Avramov, V. Gasharov, I. Peeva, Complete intersection dimension. Inst. Hautes Études Sci. Publ. Math. 86 (1997), 67-114. Zbl 0918.13008 MR 1608565

[7] L. Avramov, S. Iyengar, C. Miller, Homology over local homomorphisms. Amer. J. Math., to appear; http://arxiv.org/abs/math.AC0312412.

[8] W. Bruns, J. Herzog, Cohen-Macaulay rings. Revised ed., Cambridge Stud. Adv. Math. 39, Cambridge University Press, Cambridge 1998. Zbl 0909.13005 MR 1251956

[9] H. Cartan, S. Eilenberg, Homological algebra. Princeton Mathematical Series 19, Princeton University Press, Princeton, NJ, 1956. Zbl 0075.24305 MR 0077480

[10] L. Christensen, Semi-dualizing complexes and their Auslander categories. Trans. Amer. Math. Soc. 353 (2001), 1839-1883. Zbl 0969.13006 MR 1813596

[11] L. Christensen, Gorenstein dimensions. Lecture Notes in Math. 1747, Springer-Verlag, Berlin 2000. Zbl 0965.13010 MR 1799866

[12] W. Dwyer, J. P. C. Greenlees, Complete modules and torsion modules. Amer. J. Math. 124 (2002), 199-220. Zbl 1017.18008 MR 1879003

[13] W. Dwyer, J. P. C. Greenlees, S. Iyengar, Duality in algebra and topology. Adv. Math., to appear.

[14] H.-B. Foxby, Bounded complexes of flat modules. J. Pure Appl. Algebra 15 (1979), 149-172. Zbl 0411.13006 MR 0535182

[15] H.-B. Foxby, S. Iyengar, Depth and amplitude of unbounded complexes. In Commutative algebra and its interaction with algebraic geometry (Grenoble-Lyon, 2001), Contemp. Math. 331, Amer. Math. Soc., Providence, RI, 2003, 119-137.Zbl 02046147 MR 2013162

[16] J. P. C. Greenlees, P. May, Derived functors of $I$-adic completion and local homology. J. Algebra 149 (1992), 438-453. Zbl 0774.18007 MR 1172439

[17] R. Hartshorne, Residues and duality. Lecture Notes in Math. 20, Springer-Verlag, Berlin, New York 1966. Zbl 0212.26101 MR 0222093

[18] M. Hopkins, Global methods in homotopy theory. In Homotopy theory (Durham, 1985), London Math. Soc. Lecture Note Ser. 117, Cambridge University Press, Cambridge 1987, 73-96. Zbl 0657.55008 MR 0932260 
[19] B. Iversen, Amplitude inequalities for complexes. Ann. Sci. École Norm. Sup. (4) 10 (1977), 547-558. Zbl 0433.13003 MR 0568903

[20] S. Iyengar, S. Sather-Wagstaff, G-dimension over local homomorphisms. Applications to the Frobenius endomorphism. Illinois J. Math. 48 (2004), 241-272. Zbl 02060310 MR 2048224

[21] D. Jorgensen, L. Şega, Nonvanishing cohomology and classes of Gorenstein rings. Adv. Math. 188 (2004), 470-490. Zbl 02116574 MR 2087235

[22] J. Lipman, Lectures on local cohomology and duality. In Local cohomology and its applications (Guanajuato, 1999), Lecture Notes Pure Appl. Math. 226, Marcel Dekker, New York 2002, 39-89. Zbl 1011.13010 MR 1888195

[23] S. MacLane, Homology. Grundlehren Math. Wiss. 114, Springer-Verlag, Berlin 1967. Zbl 0133.26502 MR 0349792

[24] A. Neeman, The chromatic tower of $\mathscr{D}(R)$. Topology 31 (1992), 519-532. Zbl 0793.18008 MR 1174255

[25] P. Roberts, Le théorème d'intersection. C. R. Acad. Sc. Paris, Sér. I 304 (1987), 177-180. Zbl 0609.13010 MR 0880574

[26] P. Roberts, Homological invariants of modules over commutative rings. Sem. Math. Sup. 72, Presses de l'Université de Montréal, Montreal, Que., 1980. Zbl 0467.13007 MR 0569936

[27] J. Rickard, Derived categories and stable equivalences. J. Pure Appl. Algebra 61 (1989), 303-317. Zbl 0685.16016 MR 1027750

[28] R. Thomason, The classification of triangulated subcategories. Compositio Math. 105 (1997), 1-27. Zbl 0873.18003 MR 1436741

[29] J.-L. Verdier, Des catégories dérivées des catégories abéliennes. Astérisque 239, 1996. Zbl 0882.18010 MR 1453167

Received March 2, 2005

W. Dwyer, University of Notre Dame, Notre Dame, IN 46556, U.S.A.

E-mail: dwyer.1@nd.edu

J. P. C. Greenlees, Pure Mathematics, Hicks Building, University of Sheffield, S3 7RH, U.K.

E-mail: j.greenlees@shef.ac.uk

S. Iyengar, Department of Mathematics, University of Nebraska, Lincoln, NE 68588, U.S.A.

E-mail: iyengar@math.unl.edu 